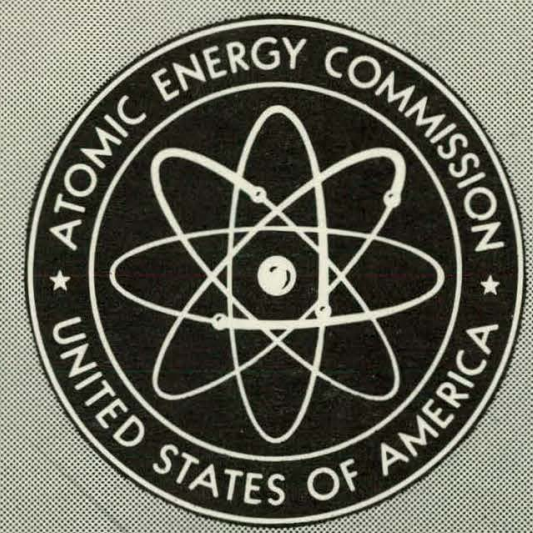

GEAP-4032

\title{
VIBRATIONAL COMPACTION OF URANIUM
} DIOXIDE

By

W. R. DeHollander

March 1, 1962

Vallecitos Atomic Laboratory

General Electric Company

San Jose, California 


\section{DISCLAIMER}

This report was prepared as an account of work sponsored by an agency of the United States Government. Neither the United States Government nor any agency Thereof, nor any of their employees, makes any warranty, express or implied, or assumes any legal liability or responsibility for the accuracy, completeness, or usefulness of any information, apparatus, product, or process disclosed, or represents that its use would not infringe privately owned rights. Reference herein to any specific commercial product, process, or service by trade name, trademark, manufacturer, or otherwise does not necessarily constitute or imply its endorsement, recommendation, or favoring by the United States Government or any agency thereof. The views and opinions of authors expressed herein do not necessarily state or reflect those of the United States Government or any agency thereof. 


\section{DISCLAIMER}

Portions of this document may be illegible in electronic image products. Images are produced from the best available original document. 


\section{LEGAL NOTICE}

This report was prepared as an account of Government sponsored work. Neither the United States, nor the Commission, nor any person acting on behalf of the Commission:

A. Makes any warranty or representation, expressed or implied, with respect to the accuracy, completeness, or usefulness of the information contained in this report, or that the use of any information, apparatus, method, or process disclosed in this report may not infringe privately owned rights; or

R. Assumes any liahilities with resnest to the use of. or for damages resulting from the use of any information, apparatus, method, or process disclosed in this report.

As used in the above, "person acting on behalf of the Commission" includes any employee or contractor of the Commission, or employee of such contractor, to the extent that such employee or contractor of the Commission, or employee of such contractor prepares, disseminates, or provides access to, any information pursuant to his employment or contract with the Commission, or his employment with such contractor.

This report has been reproduced directly from the best available copy.

Printed in USA. Price $\$ 1.75$. Available from the Office of Technical Services, Department of Commerce, Washington 25, D. C. 


\section{PAGES $\mathrm{i}$ to $\mathrm{ii}$ WERE INTENTIONALLY LEFT BLANK}


GEAP-4032

METALS, CERAMICS, AND MATERIALS

VIBRATIONAL COMPACTION

of

URANIUM DIOXIDE

by

W. R. Leitoclander.

W. R. DeHollander

March 1, 1962

U.S. Atomic Energy Commission

Contract No. AT (04-3)-36I

Approved:
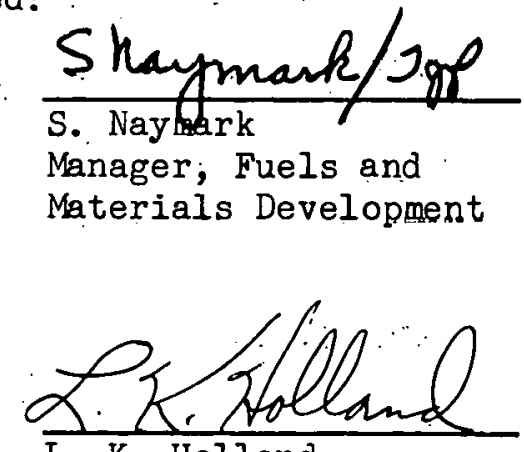

L. K. Holland

Project Engineer.

Atomic Power Equipment Department

Vallecitos Atomic Laboratory

GENERAL ELECTRIC COMPANY

San Jose, California 
PAGE NO.

LIST OF ILLUSTRATIONS v v

LIST OF TABLES vi

SUMMARY vii

RESUL'TS AND CONCLUSIONS viii

SECTIUN I INTRONICTTON $\quad l=1$

SECTION TT DESCRIPTION OF EQUIPMENT $2-1$

2.1 Primáry Equipment $2-1$

2.2 Accessory Jigs and Fixtures 2-3

SECTION III PACKING OF SINGLE-SIZE FRACTIONS I

SECTION IV BINARY MIXTURES : 4-1.

SECTION V TERNARY SYSTEMS $5-1$

SECTION VI ARRANGEMENT OF POWDER SIZES

SECTION VII QUATERNARY AND HIGHER SYSTEMS 7-1

SECTION VIII SEGREGATION · $\cdot$ 8-1

SECTION IX POWDER UTILIZATION 9-1

SECTION $X$ LOADING AND FILLING TECHNIQUE

10.1 Prellminary Blending $10-2$

10.2 Filling Procedure 10-4

SECTION XI POWDER WORKABILITY OR FRIABILITY 11-1

11.1 Experimental Procedure and Results 11-1

11.2 Evaluation of Experiment 1l-4

SECTION XII RECOMMENDA'I'LUNS HÜK FURTHER WORK

APPENDIX $\quad A-1$

REFERENCES 


\section{LIST OF ILLUSTRATIONS}

Figure No.

17

18

19

2

1

16

3

4

6

14

20

5

21

7

8

9

22

10

15

11

12 $\underline{\text { Title }}$

Binary Diagram of Ternary System

Binary Diagram of Ternary System

Binary Diagram of Ternary System

Cutaway of Typical Exciter

Electromagnetic Vibration Equipment

$\mathrm{UO}_{2}$ Particle System, The

6/8 Mesh in Binary Combinations

6/8 Mesh in Binary Combinations vs "Lumped" Efficiency

$6 / 8,8 / 10$ Binary

$6 / 8,8 / 10,10 / 14$ and Jet Fines Binary

6/8, 8/10, 35/48 Ternary Diagram

$6 / 8,10 / 14$, Binary

$6 / 8,10 / 14,35 / 48$ Ternary Diagram

$6 / 8,14 / 20$ Binary

$6 / 8,35 / 48$ Binary

$6 / 8,48 / 65$ Binary

$6 / 8,48 / 65$ Binary

$8 / 10,10 / 14,35 / 48$ Ternary Diagram

$8 / 10,48 / 65$ Binary

$8 / 10,-200$ Binary

10/14, 65/100 Binary

10/14, -200 Binary
Page No.

$7-2$

$7-2$

$7-2$

$2-5$

$2-4$

$5-3$

$4-2$

$4-3$

$4-5$

$4-8$

$7-4$

4-5

$7-5$

$4-5$

4-6

$4-7$

4.-6

$7-6$

4-6

$4-8$

4-7

4-7 


\section{LIST OF TABLES}

Table No.

I

II

III

IV

V

VI
Title

Densities of Single-Size Fractions in

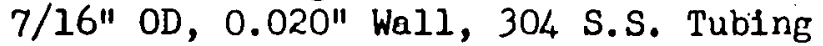

Single-Size Density Experiments

Selected Particle Compositions

Belected Particlo Donoltio日 In 304 S.S. 'l'ubing', Ủ. 4üU" Diameter'

Final Densities Predicted for Varlous

Coarse Densities (Density of Fines a.t 60 Por Cent)

Attrition of Powders During Vibration
Page No.

3-1

$3-3$

$5-4$

$5-5$

$9-1$

$11-3$ 


\section{VIBRATIONAL COMPACTION OF URANIUM DIOXIDE}

\section{SUMMARY}

1. Rough, angular particles of fused uranium dioxide of mesh size ranges defined by Tyler screen sizes the square root of two apart in diameter, will pack to densities between 58-65 per cent of theoretical in tubes close to one-half inch internal diameter or smaller.

2. Binary mixtures of 2 size ranges are capable of producing much higher densities, with one, an $8 / 10,-200 \mathrm{mix}$, yielding 82 per cent. The density achieved is directly related to the separation in size.

3. Making the coarse component of a binary mix pack to higher density is resporisible for a significant increase in density. A 3-size range of coarse, together with 5 micron powder, went to 87.8 per cent.

4. Ternary mixtures are required to get greater than 85 per cent on a routine basis. Such mixturos are easily capable of 88 per cent.

5. Quarternary mixtures or higher are probably the only approach to the consistent achievement of greater than 90 per cent.

6. The mast 1mportant tactor is the size difference between particles.

7. Segregation can be controlled and minimized by the use of noise during feeding and slight restraint during sine wave cycling.

8. Plastlc deformation $\underline{1 s}$ involved in packing $\mathrm{UO}_{2}$ since mixturés above 85 per cert are removed with difficulty from their containing tubes.

9. Powder stress is variable from lot to lot and constitutes an important variable, since the friabllity determines the best starting formula for a given final density. 


\section{RESULTS AND CONCLUSIONS}

Using high density uranium dioxide powder particles such as are obtained from arc-fused powder, consistent densities in the range of $88 \pm$ 1 per cent of theoretical density can be consistently and economically obtained if certain rules of combination of sizes are followed. In general, at least three sizes of particles are required, labeled coarse, modium, and fine, in the weight ratios varying around the values of 60 per cent coarsè, 20 per cunt medium, and 20 per cent fines. The size ratio is roughly 1 in the order of $1: 8,64$, but many variulions are possiblo.

Economical and complete utilization of powder appears probable, since there are many formulas by which such densities may be obtained. The appendix contains a listing of several hundred formulas and the densities obtained. 
SECTION I

\section{INIRODUCTION}

The concept of simply pouring uranium dioxide into a tube and sealIng it off for use as a nuclear fuel has a certain intriguing sound to it which has prompted many people to try simple powder compaction as a way of making ceramic fuel. However, without special preparation these tubes will be only about 50-70 per cent of theoretical density (TD), depending on what kind of powder is used. This low density leads to certain penalties in use. First, an enrichment penalty must be paid because higher enrlchment is required to get the necessary reactivity. Second, in power reactors, enough heat is generated in the fuel to cause extensive sinterIng which causes the fuel to lump together in isolated clumps of high density, which affects the reactor performance.

These effects can be mitigated to some extent by initially packing the fuel to higher densities.

It is the object of this document and the work described therein to explore some of the criteria which are necessary to pack uranium dioxide to higher densities in the tubes of final use by the appilication of sonic vlbration. Such fuel can be economically produced and is a satisfactory substitute lor the standard pelletized fuel. 


\section{THIS PAGE}

WAS INTENTIONALLY

LEFT BLANK 
SECTION II

\section{DESCRIPTION OF EQUIPIENT}

\subsection{Primary Equipment}

$\therefore \quad$ The equipment used in this investigation consisted of an MB Corporation Model C-10 electrodynamic vibration exciter rated at 1200 force-pounds, driven by an MB Model T35IMC power amplifier and control console, complete with oscillator, automatic cycling, and vibration meter. The C-10 exciter can be driven to a l" peak-to-peak displacement or $70 " /$ second velocity or $68 \mathrm{G}$ 's acceleration or 27 amperes in the driver coil. Any one or more of these parameters may be the limiting factor in performance. The electrodynamic system was chosen because precise control of conditions of vibration is possible. The specimen mounting table is cast magnesium and contains a pattern of stajn]ess steel inserts for specimen mounting. There is incorporated within the shaker a velocity coil which permits monitoring the table motion.

The T351 MC power amplifier and control console supplies 12,000 watts plate dissipation from a total of 25,000 watts input. However, power for the field also comes from the console. The console is provided with an automatic cycling oscillator which has a useful range of 5-5000 cps on one position and 5005 to 10,000 cps in the second position. It is provided with circuitry which can maintain constant acceleration or displacement while the frequency is varied. The frequency can be varied by a motor-driven capacitor which rotates 
the dial at a given number of degrees per minute. Thus sweep rates are adjustable to require from one minute to 100 minutes to sweep from 10 to $2000 \mathrm{cps}$. Any desired portion of the frequency band may be selected for sweep. The built-in level control has correction rates of $10,20,40$, or 80 decibels per second.

In the particular setup as used at $A P E D$, the basic equipment has been moditied in the tollowing ways:

1. A bank of 5 HP2OOAB oscillators has been added.

2. These oscillators feed a Bogen model MXM mixer.

3. The output of the mixer or of the cycling oscillator may be selected at will to be fed to the power amplifier.

4. The input signal may be half-wave negative or positive or pure sine wave.

5. The mixer, oscillator and sweep speed, as well as amplitude control, are remotely connected so that an operator at the loading box can adjust the parameters of vibration frequency and amplitude.

The bank of five oscillators provides for selecting any combination of exciting frequencies to be simultaneously fed to the exciter. Thus several rod resonances may be simultaneously excited if this is deemed necessary or desirable. Even the cycling oscillator can be blended with other frequencies at will. A remote vibration weter at the loading station allows close observation of displacement, velocity, or acceleration. Plug-in jacks have been installed in order to be 
able to observe the wave form at several positions. A set of three cathode followers will accept the input from three accelerometers for display on a Tektronic dual trace oscilloscope. A Slip-Sync system, model D, manufactured by the Chadwick-Helmuth Company, permits slow motion study, motion pictures, and direct observation to be employed. This latter equipment is useful only with pure sine waves and hence, in the latter stages of the program, has been of little use. Initially, it was quite beneficial in establishing just which modes were being excited.

Photographs of the console, shaker, and accessory equipment illustrate their general characteristics. (See Figures $I$ and 2.)

\subsection{Accessory Jigs and Fixtures}

Before rods can be filled with uranium oxide on the shaker, means must be found for holding the rods on the table, pouring in powder, controlling dusting and contamination, positioning operators and glove boxes at desirable heights. The approaches at APED have been slanted toward versatility and quick change in order to meet the demands of a broad development program. It has been deemed advisable to be able to quickly change from one diameter to another without first machining new jigs, etc. For mounting single rods on the table, a modified Jacobs Flex-a-collet chuck has been most useful and when properly used is quite satisfactory. The hand wheel was removed from a hand wheel type. The collet is tightened by using a drift pin and hammer against the normal hammering tips. This collet will accept diameters from $1 / 8 "$ to $1-3 / 8 "$. 


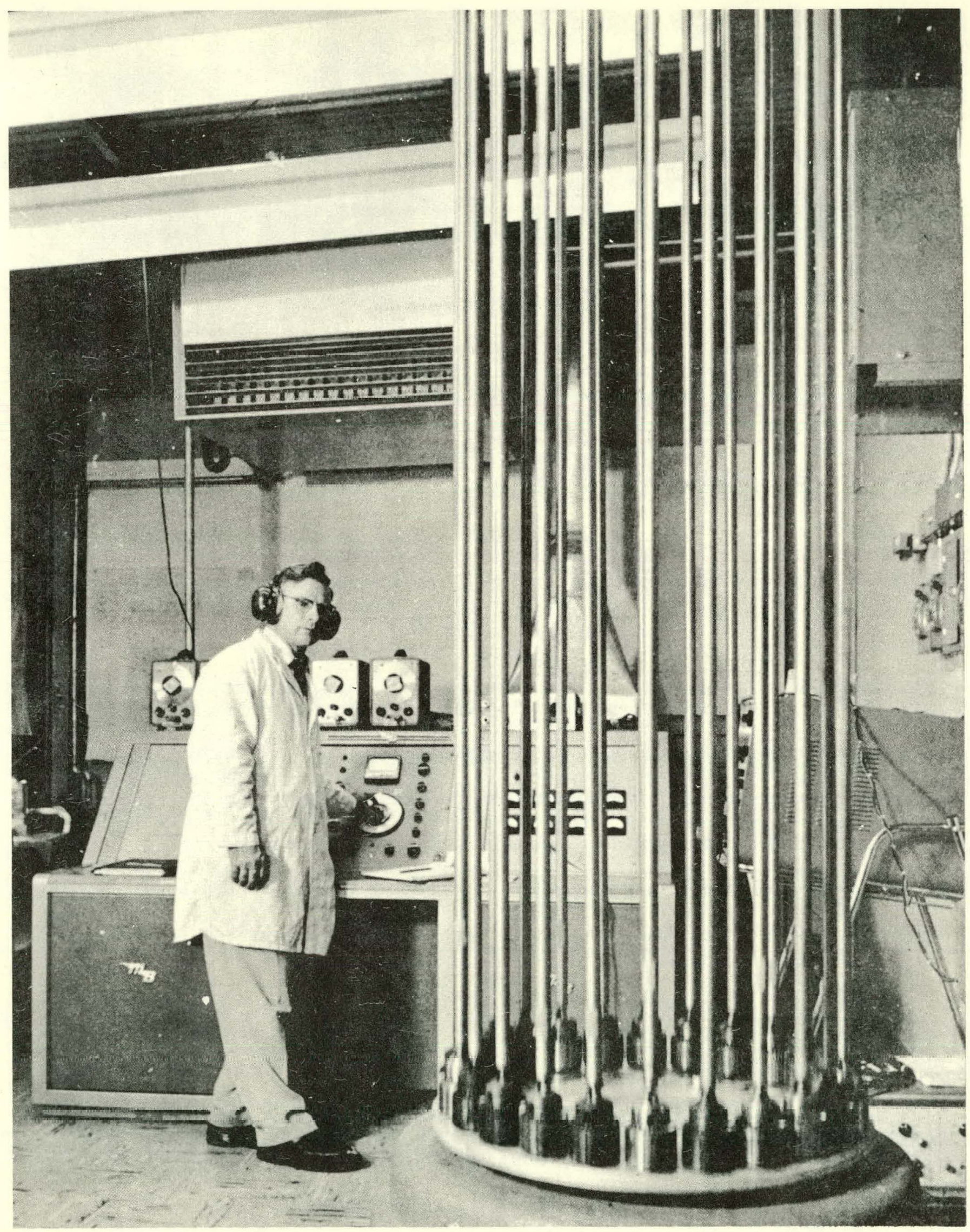

FIGURE I

ELECTRO MAGNETIC VIBRATION EQUIPMENT SHOWING JIG FOR VIBRATING 24 RODS AT ONE TIME 


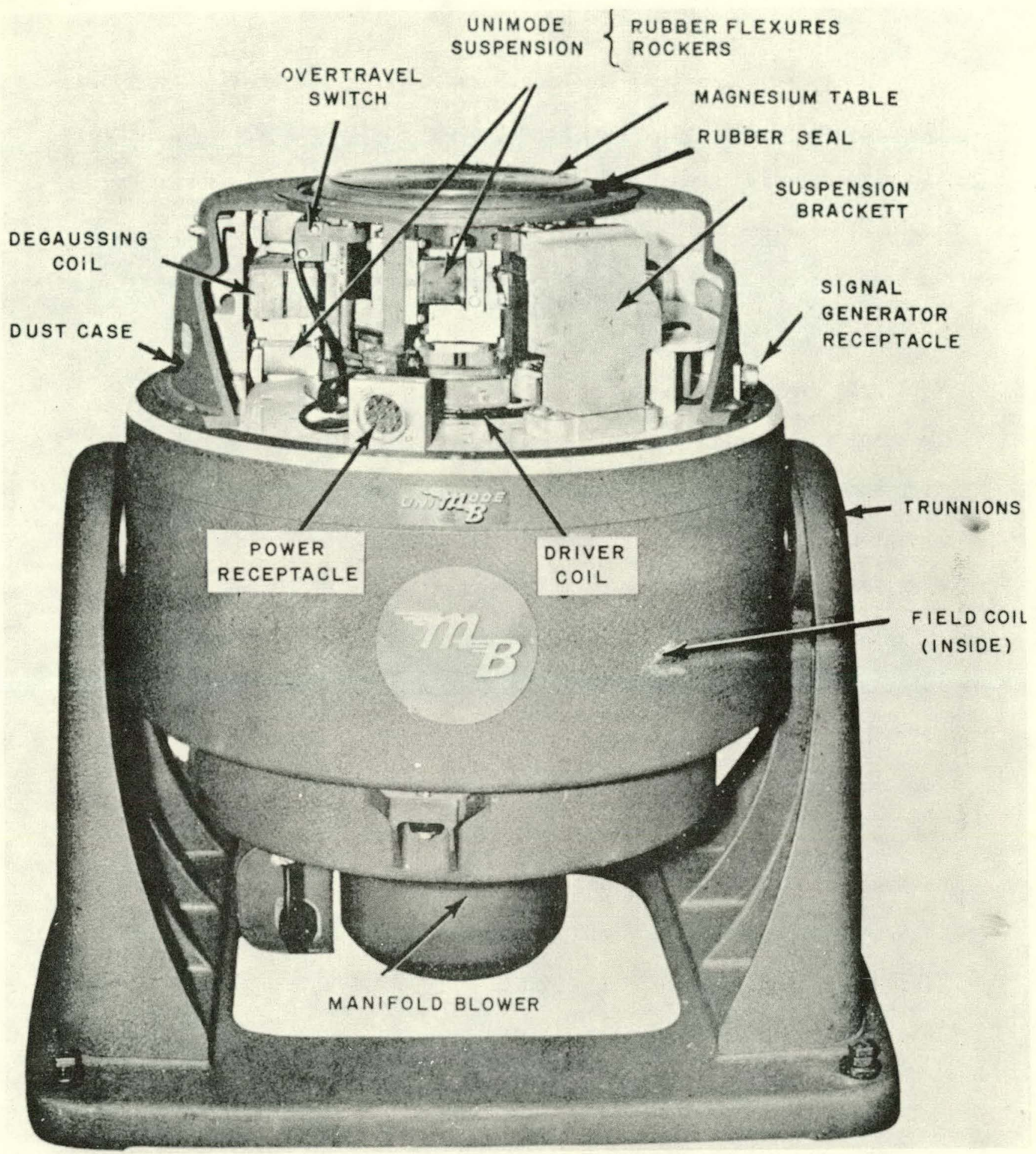

CUTAWAY OF TYPICAL EXCITER

FIGURE 2 
In order to meet the requirements of variable height of specimen, two elevators were purchased from Economy Engineering Company. One is hand operated and is used for positioning the loading box at the desirable height. The second is motor driven and positions the operator at a convenient height at the box. In this way rods from 4" high to $12^{\prime}$ high can be loaded.

The rods protrude through the bottom of the box by way of a special pipe nipple assembly locked to the box with 0 ring seals top and bottom to prevent sifting of $\mathrm{UO}_{2}$. The top of the nipple is closed with a specially molded rubber cap. The rubber cap tightly seals the rod from leakage. A specially molded 6" rubber funnel is slipped onto the protruding end of the rod. A tubular Syntron feeder with a right angle bend at the end protrudes into this funnel, loosely, with only slight clearance, to minimize dusting. The Syntron feeders are equipped with hoppers for holding a proviously weighod chargo of powder. They are operated by a master switch and a Variac which allows variable level operation to ensure complete emptying of the charge. Directly above each tube opening is a pneumatic cylinder which can apply pressure to a rod which presses on the powder during the final stages of compaction. In the event that a charge of powder is too large for filling the tube correctly, a small suction hose and catch bottle allow removal of the powder from the upper part of the funnel so that the system can be opened without spillage. 


\section{PACKING OF SINGLE-SIZE FRACTIONS}

The first starting point was to determine how rough particles of arc-fused $\mathrm{UO}_{2}$ of narrow steve size range packed all by themselves in the tubing of Interest. Consistent with the data of McGeary ${ }^{1}$, a dependence of the density on the tube diameter was shown, although almost all size fractions packed to densities between 58 and 65 per cent of theoretical. Table I shows one typical set of data.

\section{TABLE I.}

DENSITIES OF SINGLE-SIZE FRACTIONS IN 7/16" OD,

$0.020^{\prime \prime}$ WALL, 304 S.S, TUBING

$\left(\right.$ ID $\left.=0.4375-0.040=0.3975^{\prime \prime}\right)$

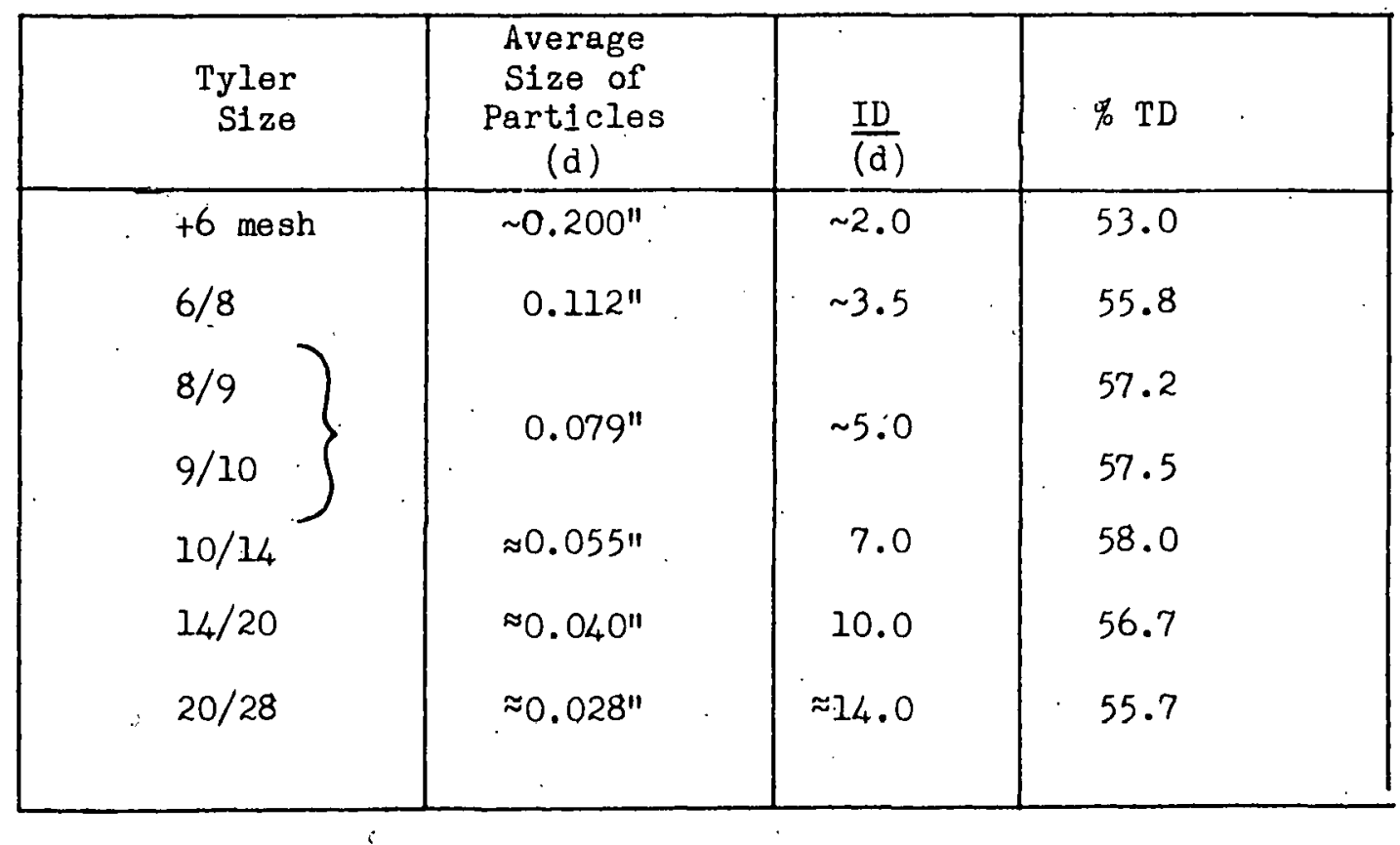


Such data as this indicates nicely how certain size particles fit best in a given tube when the size difference is quite small. The data in Table I were taken at low G levels and represent as-packed densities without working. When the $20 / 28$ fraction was worked harder and more attrition had occurred, it was worked up to a density of 62.4 per cent. However, considerable attrition had occurred at this time. Table II lists, for reference purposes, all the data on single-size fractiono that have boon run at $\Lambda$ PED. Thore ie a wide spread of tub- . ing size, sample size, and column hoight represented as well as a material quality variation in the fused materials. It should he evident. from these data that high densities will require more than one particle size, as can be deduced from the few wider-band mixes included. 
TABLE II

SINGLE SIZE DENSITY EXPERIMENTS

(Not Including Table I)

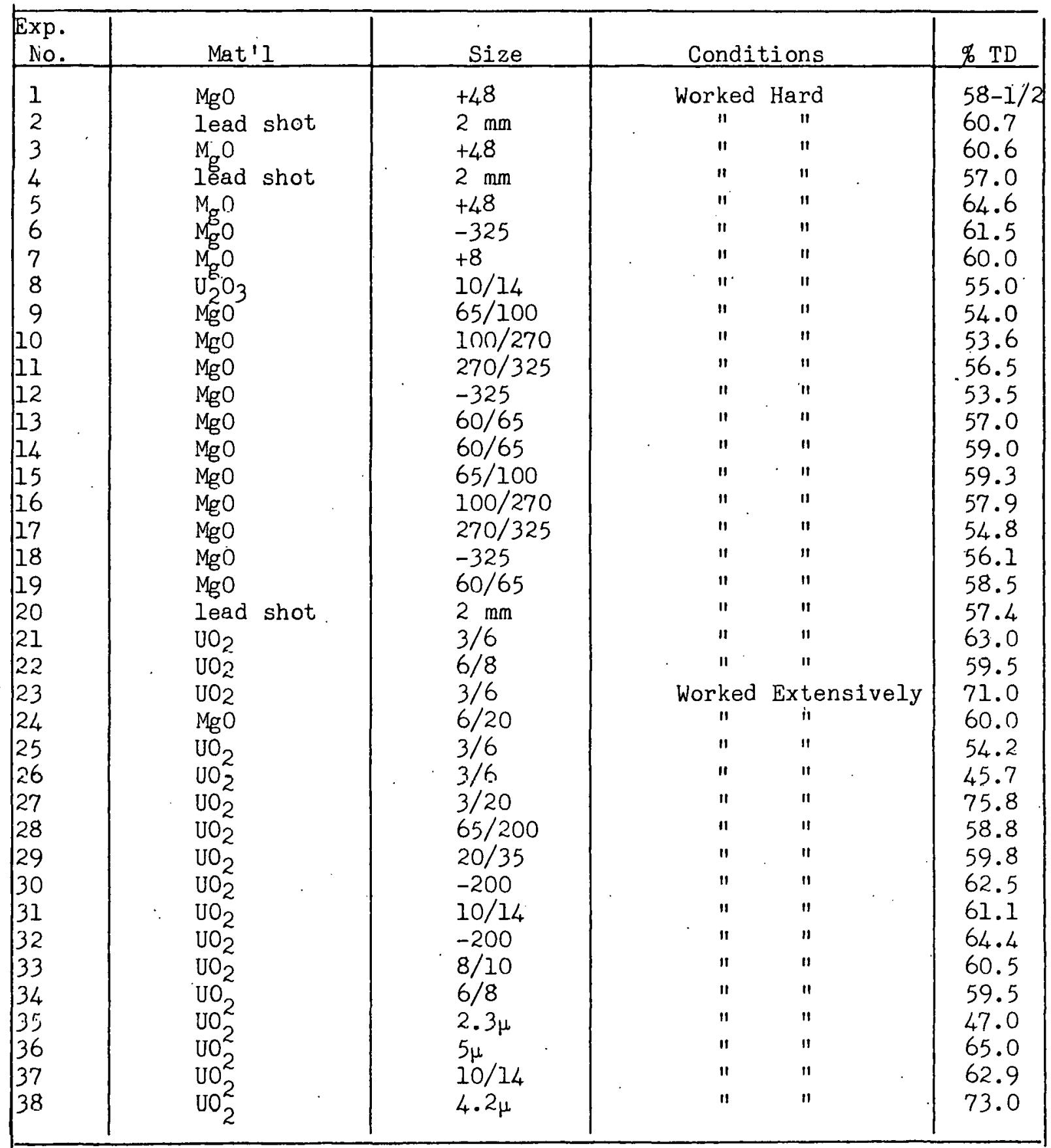


THIS PAGE

WAS INTENTIONALLY

LEFT BLANK 
SECTION IV

\section{BINARY MIXTURES}

Simple binary mixes would seem to offer a possible approach to higher densities; these higher densities have been observed by several investigators. Theoretically the density can be calculated by the formula,

$$
d_{b}=\left[\left(100-d_{1}\right) \frac{d_{2}}{100}\right]+d_{1}
$$

where $d_{b}=$ per cent TD of binary mixture

$$
\begin{aligned}
& \mathrm{d}_{1}=\text { per cent } T D \text { of flrst fraction only } \\
& \mathrm{d}_{2}=\text { per cent TD of second fraction only (if there were no wall } \\
& \text { effect). }
\end{aligned}
$$

As an example, if $6 / 8$ were to pack to 60 per cent by itself and $35 / 48$. also packed to 60 per cent by itself, then a binary maximum of these two would yield $60+0.6(40)=84$ per cent. Figure 3 is a graph1c representation of various values substituted into the equation and $d_{b}$ calculated. It would he naive to suspect that the second fraction would pack just as well in the spaces between the first size as it would all by itself in the tube. Hence one should assign an efficiency factor to the packing. Such efficiency factors can be calculated from the graph of $6 / 8$ mesh in binary combinations, Figure 4. As the size separation gets larger, the curve approaches the theoretical limt of possible density where the efficiency factor is unity. These efficiency factors which would be calculated are "lumped" efficiency factors and are simply good "fudge factors" to be used in guesstimating final densities. If however, attempts are made to 


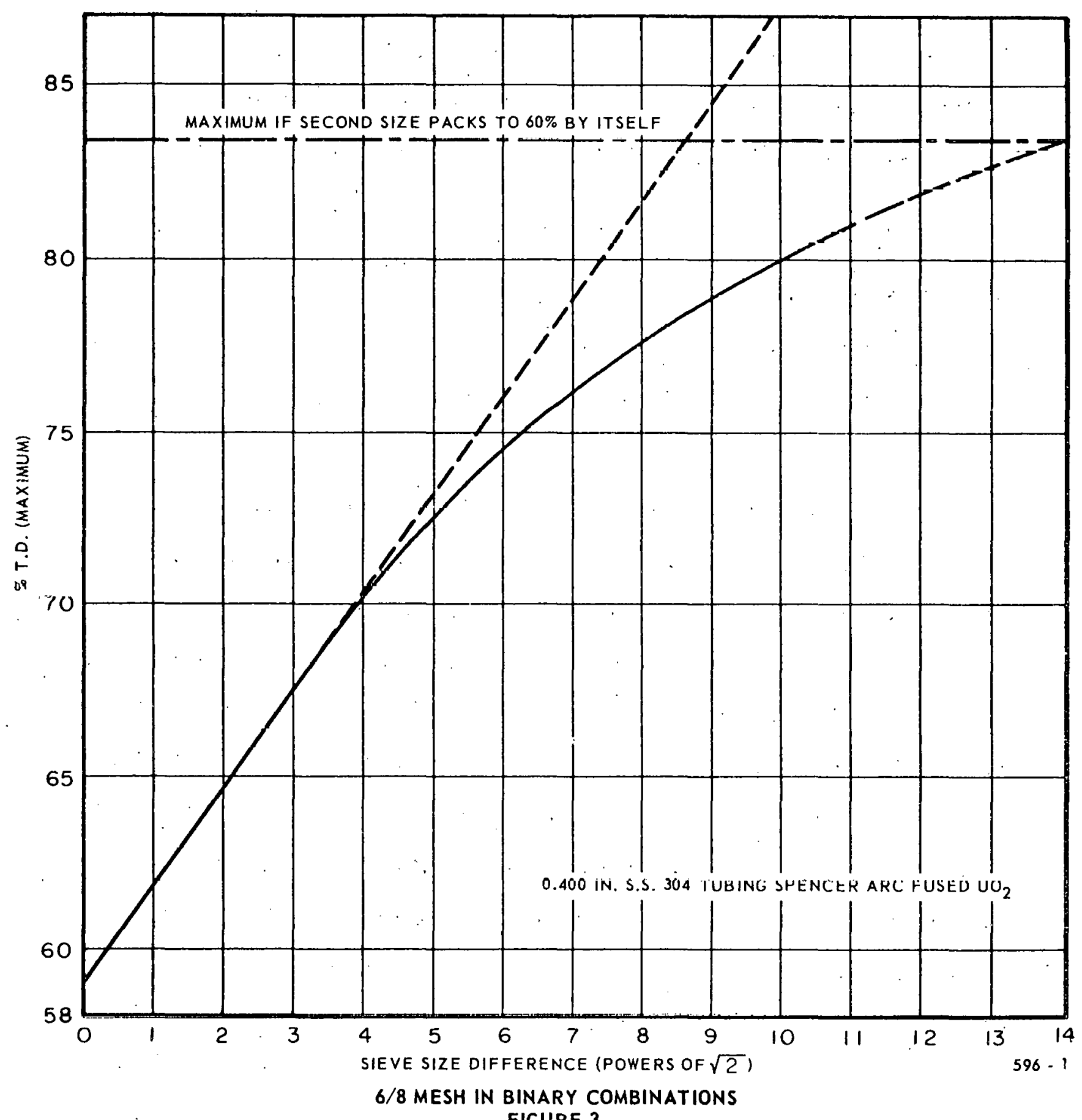

FIGURE 3 


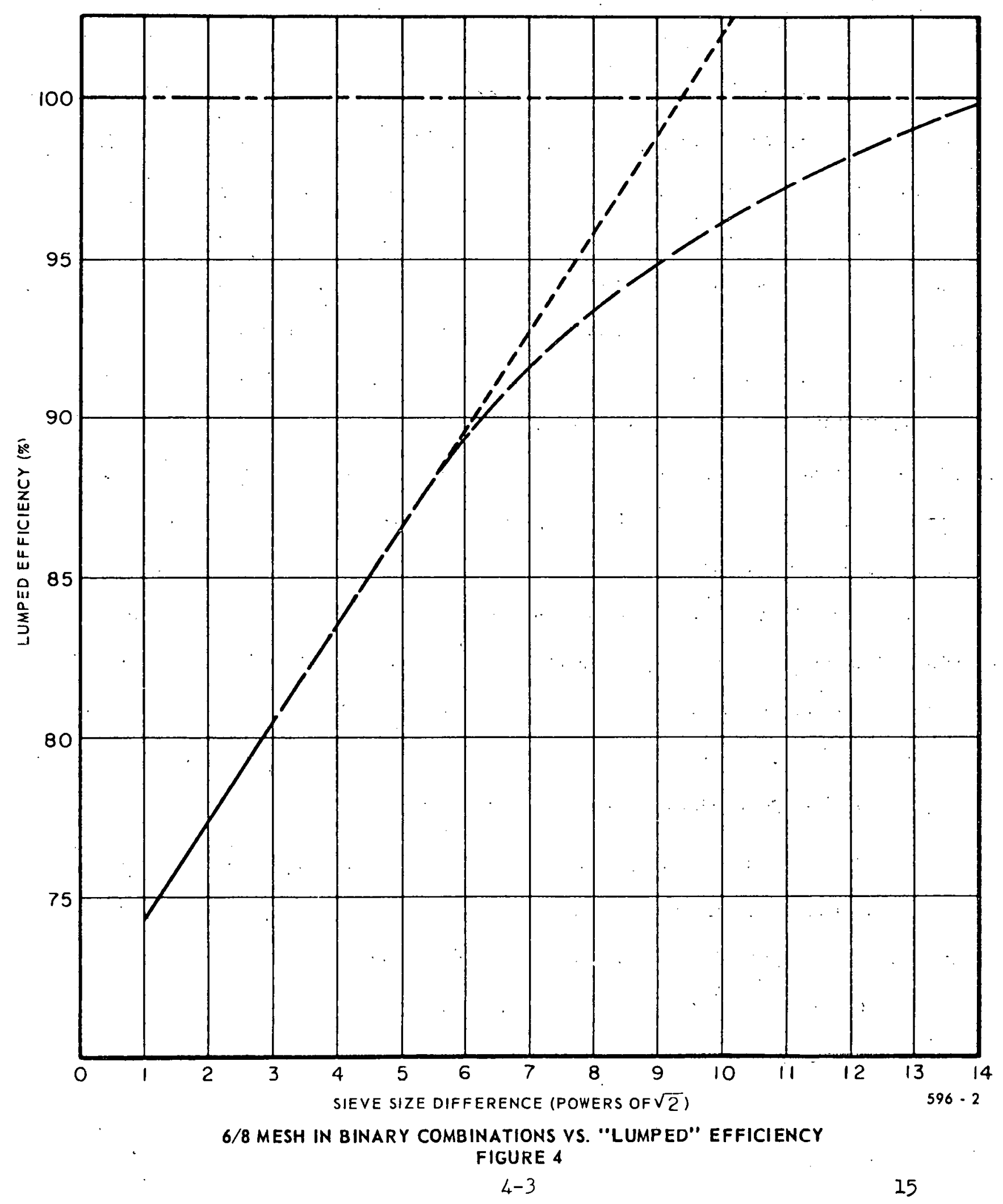


calculate the composition of the binary mix from the formula, then erroneous results are obtained. The correct formula then becomes,

$$
d_{b}=d_{1} F_{1}+\frac{F_{2} d_{2}}{100}\left(100-d_{1} F_{1}\right)=d_{1} F_{1}+d_{2} F_{2}-d_{1} d_{2} F_{1} F_{2} / 100
$$

where $F_{1}$ and $F_{2}$ are factors used to describe how much each fraction is packed, based on its performance alone. The interaction portion of the formula above essentially contains most of the problem. In the event that the coarse fraction is loaded first and is held in place so it cannot move, then $F_{1}$ can be made unity. McGeary (loc cit) did just this. However, most investigators do not add one fraction into another but load a mixture of the two and calculate the density. In this way, particles of the second phase can get between particles of the first phase and cause a change of spacing. Figures $5,6,7,8,9,10,11,12,13,14$, and 15 summarize the data on several binary systems of intereșt. The efficiency factor on the second phase varies from 40 per cent to 100 per cent. In general, the maximum for any binary system can be quickly found by running experiments from 60 per cent coarse to 75 per cent coarse. The figures quoted easily demonstrate that binary mixtures alone are worthy of attention; the density of 82 per cent has been attained using $8 / 10$ and -200 mesh. Fiven higher values are possible with fines of -200 mésh.

In the graphs of binary mixtures, the dotted line running from the coarse to the maximum is drawn between the starting density of the coarse only and the point of the calculated binary maximum. The real form of this line should be a portion of an hyperbola given by

$$
d_{b}=\frac{d_{c}}{x_{c}}
$$




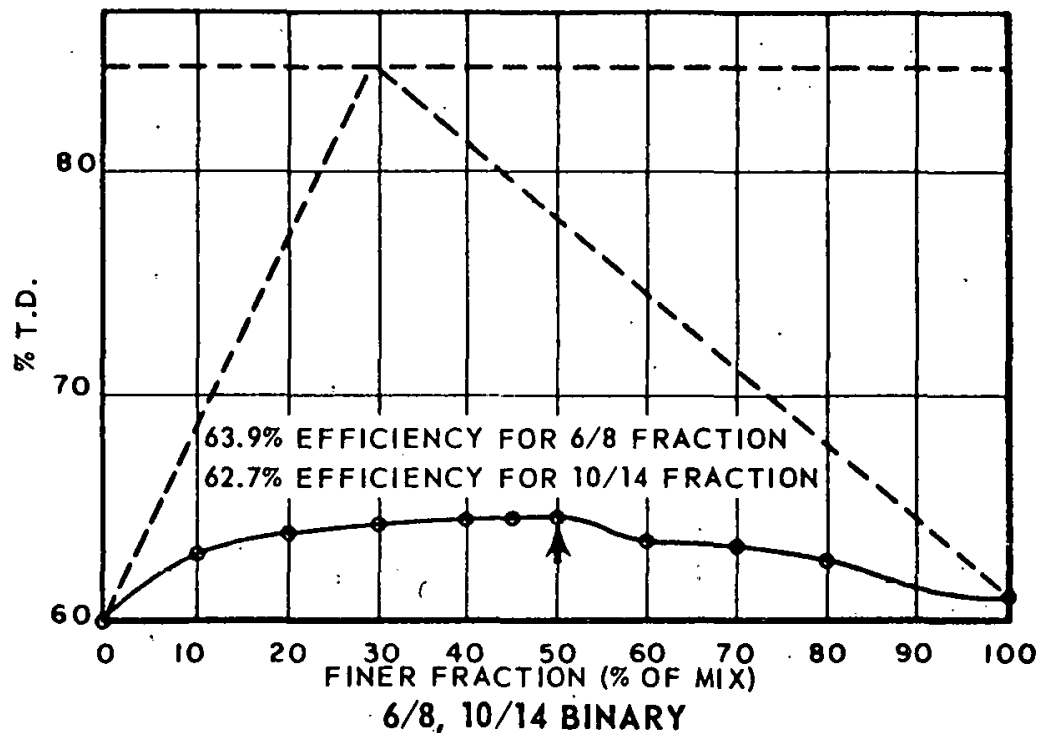

FIGURE 5

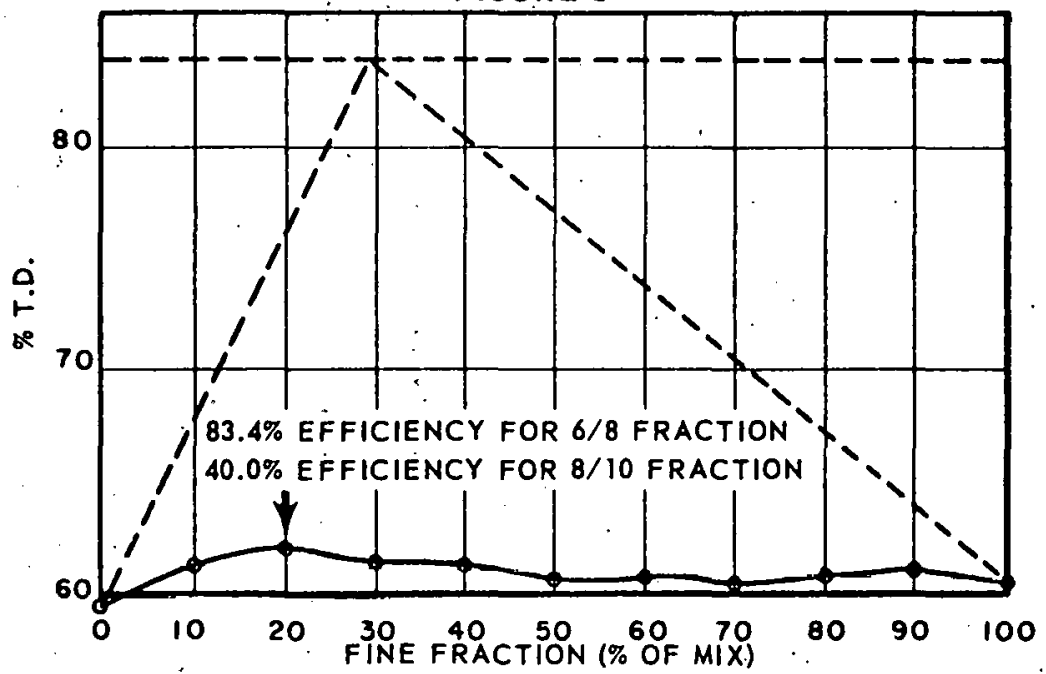

6/8, 8/10 BINARY

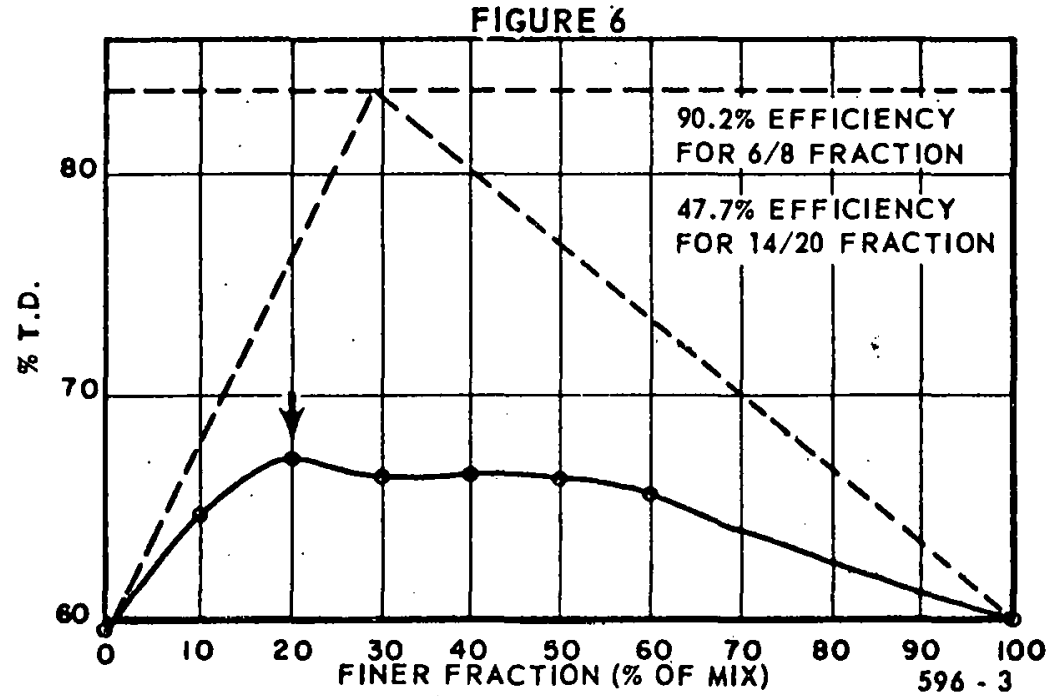

$6 / 8,14 / 20$ BINARY

FIGURE 7 


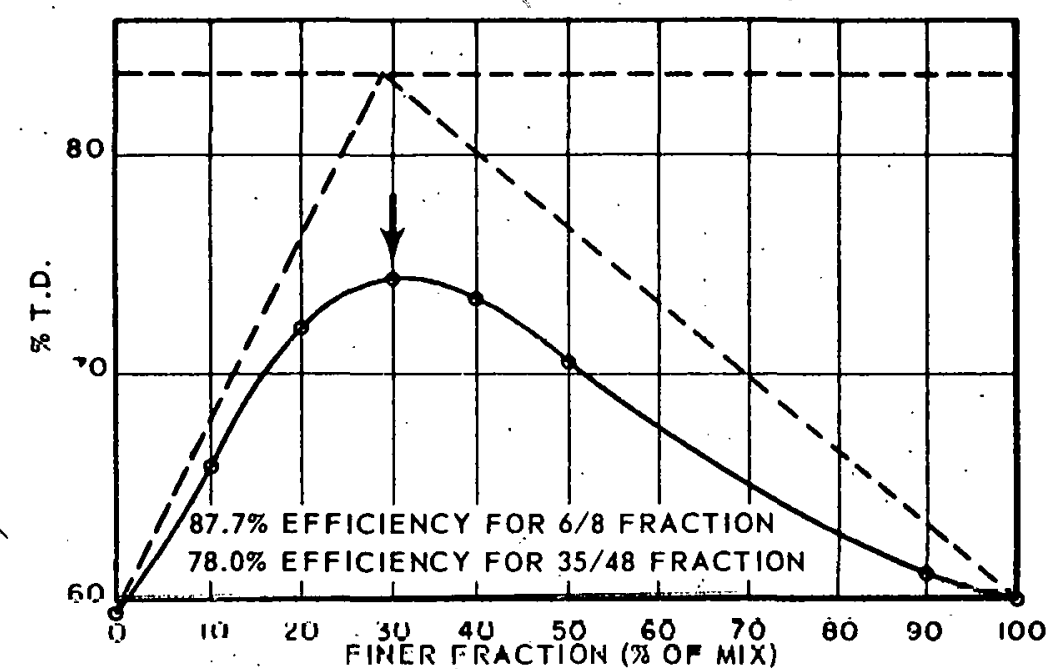

6/8, 35/48 BINARY

FIGUURF \&

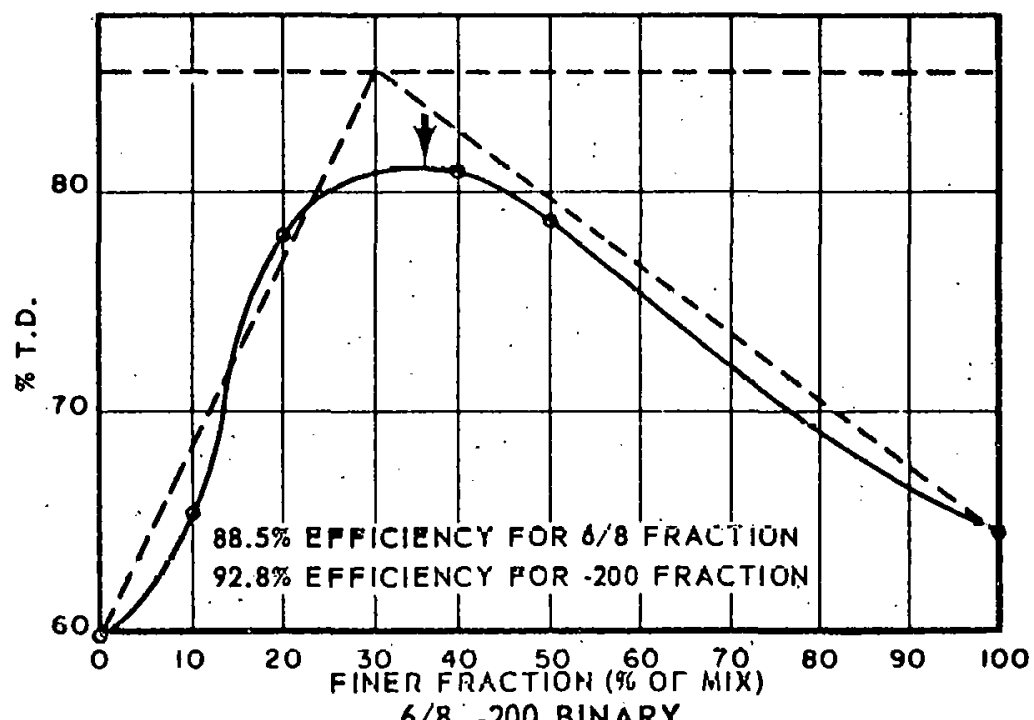
$6 / 8,-200$ BINARY

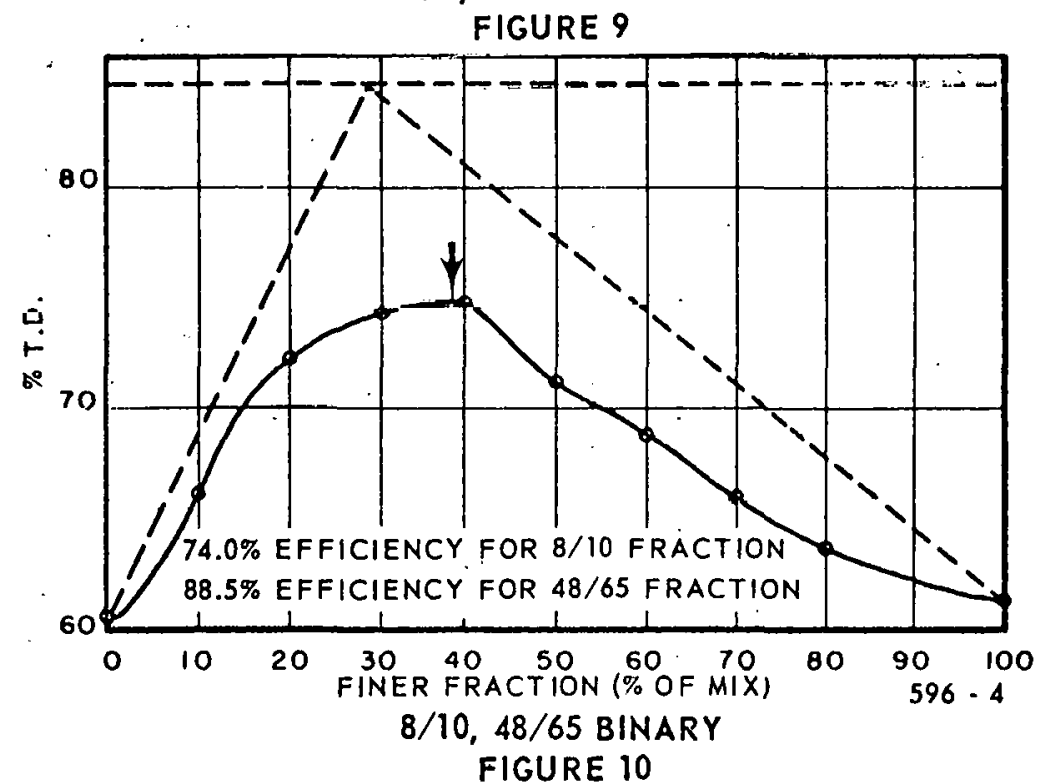

$$
\text { 4-6 }
$$




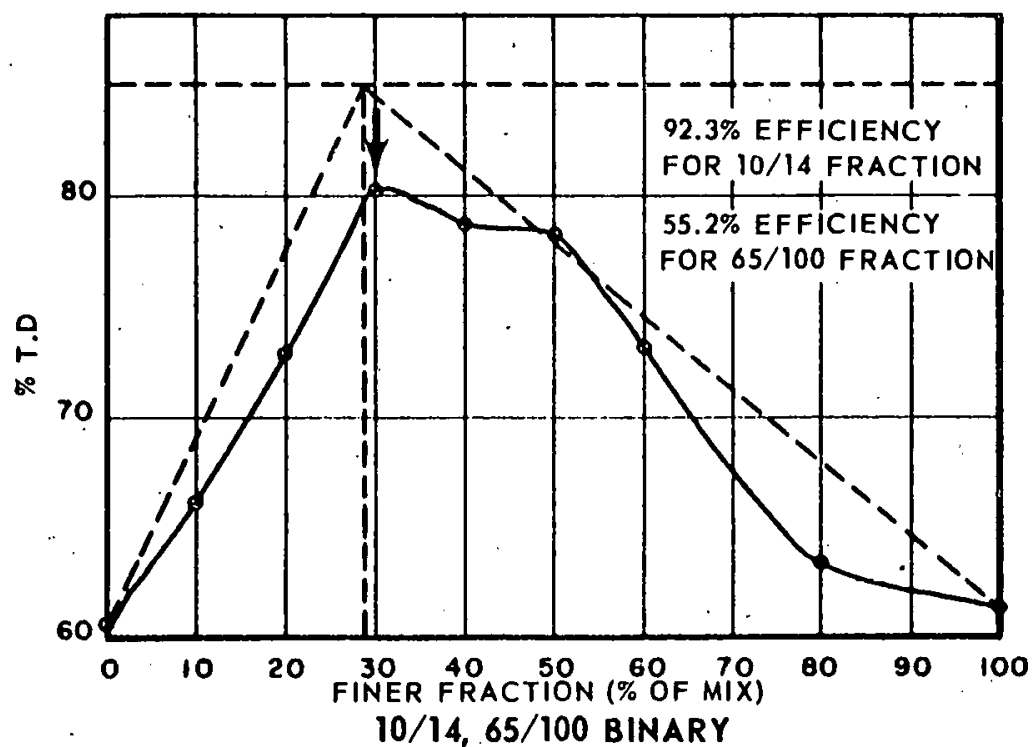

FIGURE 11

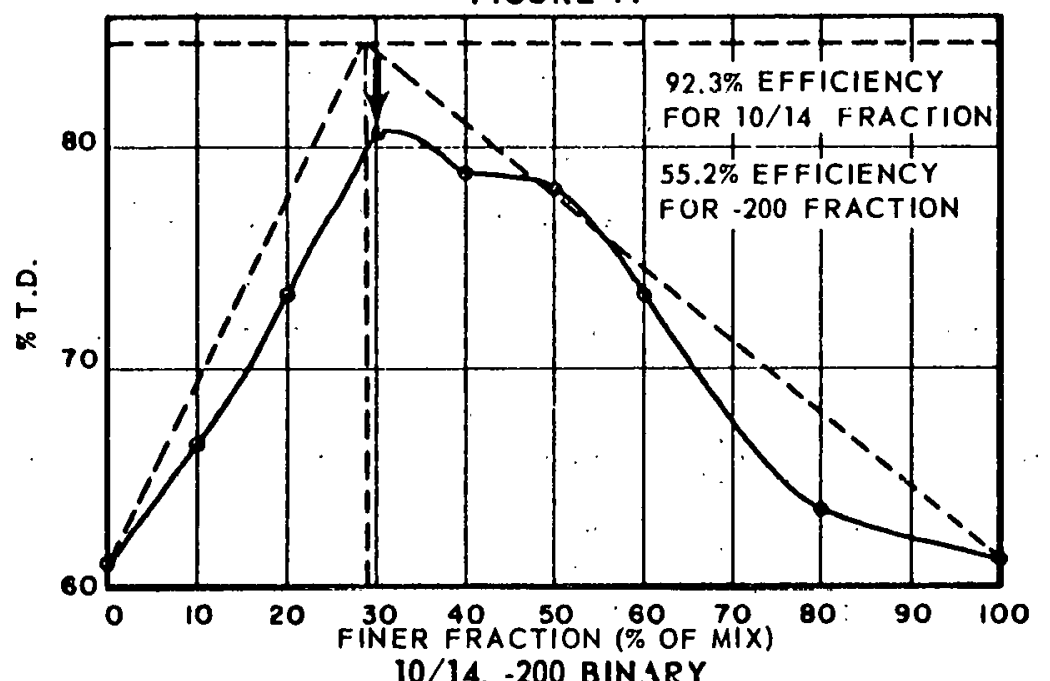

10/14, -200 BIN.ARY

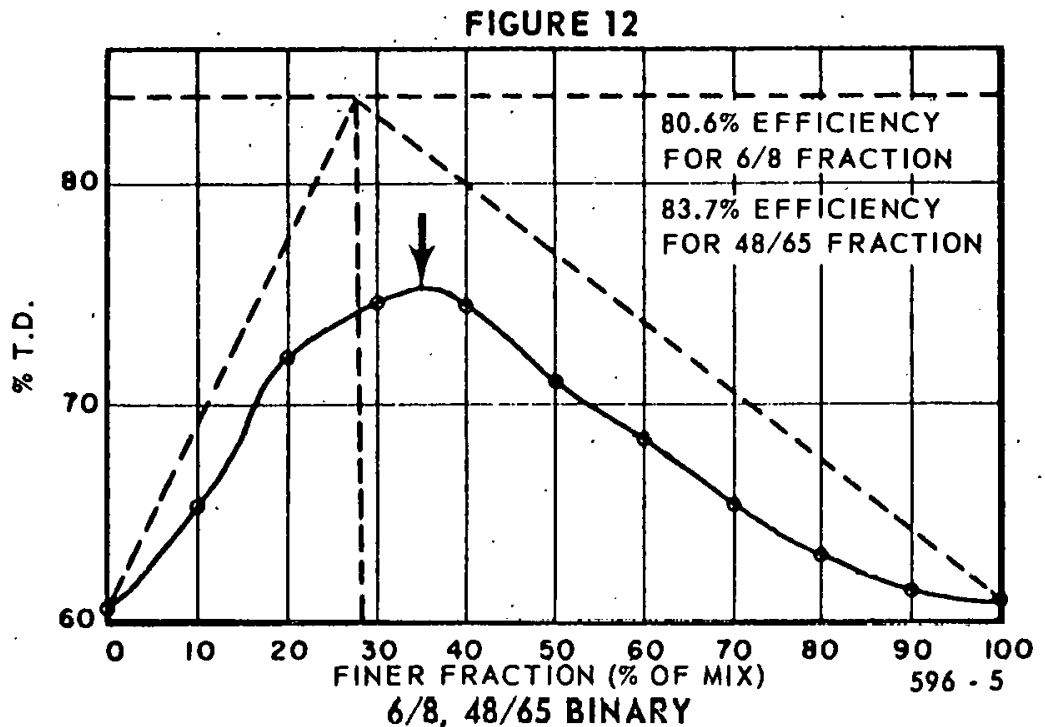

FIGURE 13 


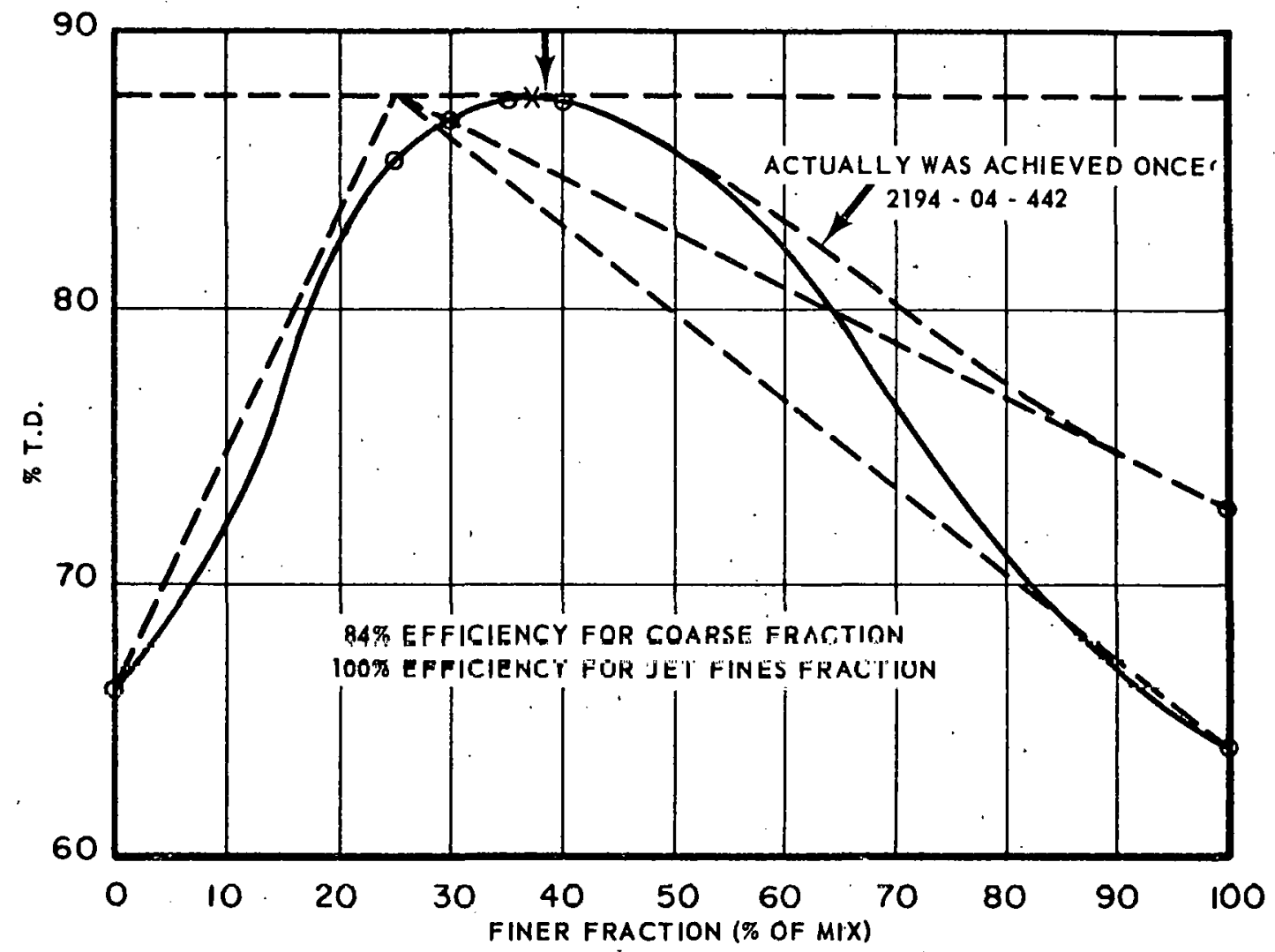

$6 / 8,8 / 10,10 / 14$ AND JET FINES BINARY FIGURE 14

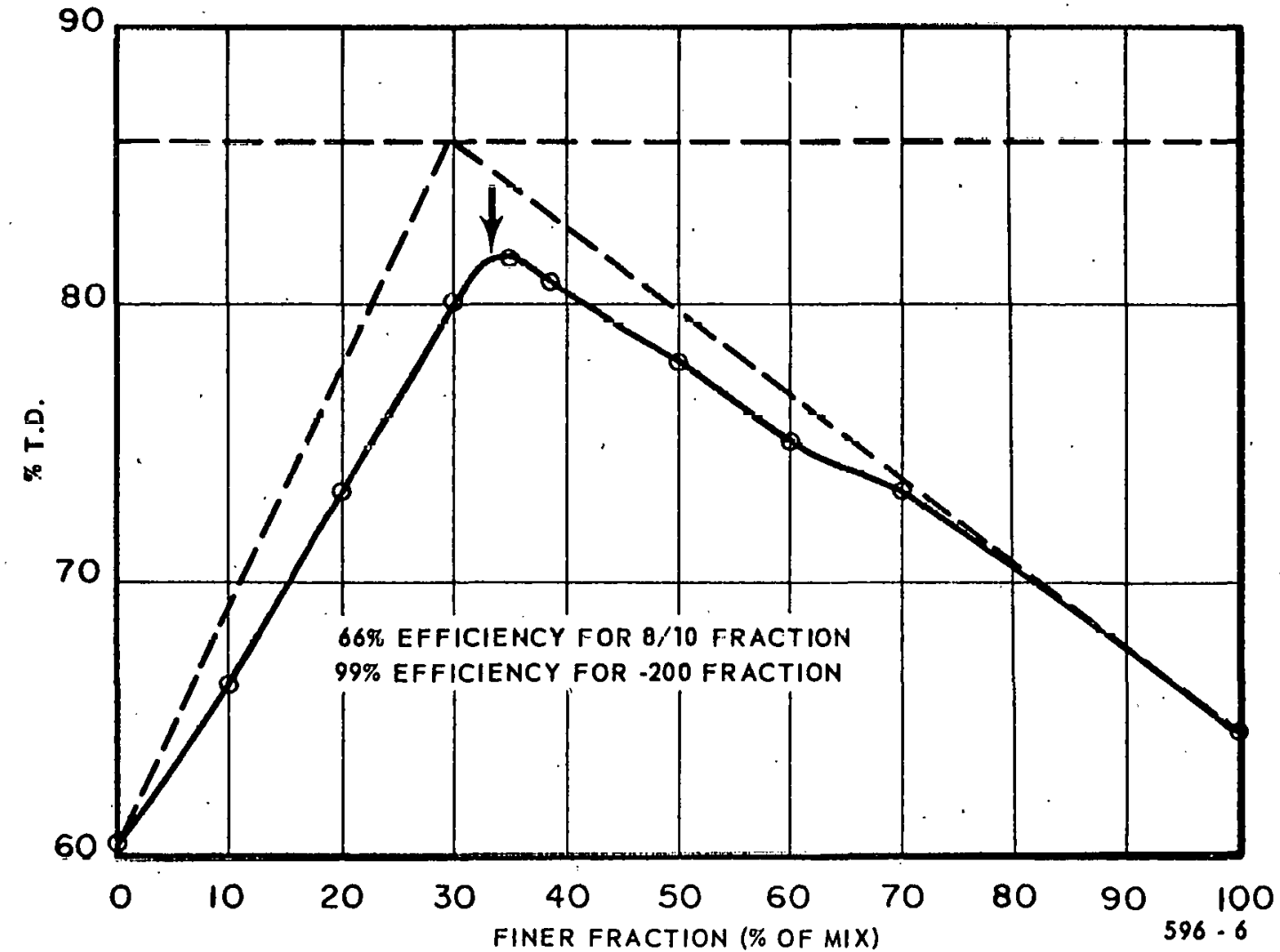

8/10, -200 BINARY

FIGURE 15 
where $d_{b}=$ density of binary mixture

$$
\begin{aligned}
& d_{c}=\text { density of coarse fraction only } \\
& x_{c}=\text { fraction of coarse particles }
\end{aligned}
$$

For practical purposes, the straight line drawn is good enough to see how well a system is performing. The equation used here 1 s based on the assumption that the volume occupied by the combination is identical to the volume occupied by the coarse alone, with the second fraction merely filling the volds between the larger particles. The equation holds for the region between the pure coarse fraction alone and the saturated binary maximum, when all the holes are just filled to the maximum possible.

On the other side of the diagram or starting from the fine fraction only, it can be assumed that each coarse particle added to the fines, "dissolves" in the fines and gives a volume increase equal to the volume of the particle alone. On this basis then the density would be

$$
\mathrm{d}=\frac{\text { ulass }}{\text { volume }}
$$

in which mass $=m_{f}+m_{c}$

$$
\text { where } m_{F} \text { is the mass or weight of fines }
$$

$$
m_{c} \text { is the mass or weight of curist. }
$$

Let $x$ refier to the fraction of each size present,

$$
x_{c}+x_{F}=1
$$

Let us also assume a normalized particle density of 1 such that

$$
\operatorname{Vol}_{c}=\frac{\operatorname{mass} c}{d}=x_{c}
$$

that is, the particles are wholly dense. Then,

$$
d_{b}=\frac{x_{F}+x_{c}}{x_{c}+\frac{x_{F}}{d_{F}}}=\frac{d_{F}}{x_{F^{\prime}}+x_{c} d_{F}}=\frac{d}{x_{F}+d_{F}-x_{F} d_{F}} \begin{aligned}
& \text { or times } 100 \text { for per } \\
& \text { oent of theoratical }
\end{aligned}
$$


The graph of this equation is also part of an hyperbola but the curvature is so slight as to be approximated by the dotted straight line.

Simultaneous solution of these two expressions for the binary density should yield the theoretical composition of the binary maximum as

$$
x_{F}=\frac{d_{F}\left(1-d_{c}\right)}{d_{c}+d_{F}-d_{C} d_{F}}
$$

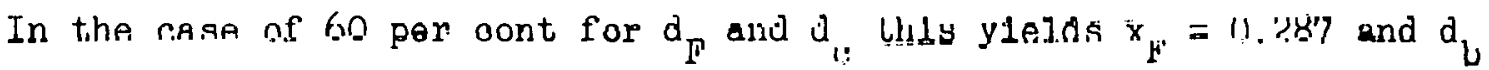
(max) as 84.2 per cent.

The equations developed above must only be expected to hold when the size difference is quite great and when only the first part of the curve is considered. It is surprising how soon the curve deviates on the fine side of the diagram. In general, the coarse side of the field is more closely adhered to; this is the basic wisdom behind the approach taken by McGeary.

As was seen in the discussion on the packing of single mesh sizes, tho ooaroc fraetion sliuili be so chosen as to f'it the geometry lo the best possible density and to use a fine fraction which also packs well by itself. The size separation between a 6 to 8 mesh fraction and a -200 mesh f'raction is quite large, large enough to make for efflulerl fitting of fines between the coarse. This 1 s also true of a 10 to 14 mesh fraction and -200. Hence if the $10 / 14$ mesh fraction packs one per cent higher in density than a $6 / 8$ mesh fraction, one could expect to see the benefit in a higher. final density. Corroborating evidence will be presented later.

It 1 s evident from the form of the equations that a one per cent benefit will not be obtained from a one per cent increase in either $d_{c}$ or $d_{F}$. For example, use of $d_{F}$ as 60 per cent and $d_{c}$ as 60 per cent yields $x_{F}$ as 
0.287 and $d_{b}(\max )$ as 84.2 per cent. Using $d_{F}$ as 60 per cent and $d_{c}$ as 61 per cent yields $x_{F}$ as 0.277 and $d_{b}(\max )$ as 84.4 per cent. Thus only a 0.2 per cent increase was seen for a change of 1 per cent in the density of the coarse fraction. Figure 3 shows a mesh size separation of one size fraction is good for at least 1 per cent improvement in density.

The binary maximum approach is probably the best basic means of achieving high densities, especially when used in a cascade manner. One set of results, presented under the discussion of ternary and higher systems, underscores the basic wisdom of the binary approach. 
THIS PAGE

\section{WAS INTENTIONALLY LEFT BLANK}


SECTION $\mathrm{V}$

\section{TERNARY SYSTEMS}

The usual maximum achleved in a pure binary system has been observed to be of the order of 82 per cent of theoretical. However, a density of 87.8 per cent was obtained with a binary mixture (Figure 14). That system was composed of a coarse fraction which, in turn, was a maximum among three adjacent mesh size ranges. It therefore constituted a quaternary. rather than a binary. However, it was composed of a coarse and a fine fraction, with no intermediates.

In order to achleve higher densities it seems necessary to add st1ll a third component which will be called the middle fraction. Systems of three particle sizes can still be plotted on graph paper if triangular. coordinates are used. Thus the study becomes quite analogous to phase. diagram studies of the physical chemist. By plotting the experimental data in this manner, a mental picture of the interactions can be obtained.

Varlous investigators $1,2,3$ have observed that a maximum can occur in a 3-phase system at only one region, situated in the vicinity of 65 per cent coorse, 15 per cent middles, and 20 per cent fines. This is the same conclusion which is drawn by theoretical considerations of fitting particles within the volds between larger particles. The analyses given by Westman and Hug $111^{2}$ and by McGeary ${ }^{1}$ illustrate the limiting case of infinite separation. In most cases the practical range of sizes is from about a $6 / 8$ mesh range down to about -325 . This barely allows a diameter 
ratio of 100:10:1 which is not quite infinite but reasonably good. Combustion Engineering people, Bruce Murtha, et al. ${ }^{4}$ feel they will approach $>90$ per cent routinely by simple three-phase mixes. In reality, in their method of compaction, they may start with three particle sizes but quickly create a multi-particle system.

In this investigation, simple three-phase systems have not yielded densities in excess of 88 per̀ cent.

Again, tho uoo of tho analytioal oxpreseione given by Westman and Hugill are quite helpful when no interaction is allowed between the various sizes of particles. The assumption that the volume is equal to the volume of only the coarse particles implies that the coarse component is in contact and therefore that the next size is fitting between the holes. The more practical system involves letting the coarse particles separate a little if need be, to accommodate the next size which may be slightly too large to fit the voids. This is a far more complicated system to study, and is probably best studied just by experiments. To this end the system of three particles consisting of $10 / 14,65 / 100,200 / 270$ was studied entirely in the field. If the 10/14 is assigned an averuge size of 0.0555 inches, the 65/100,0.070 inches, and the $200 / 270$ as 0.0023 inches, the rat1o of $1: 2.8: 22.2$ is a long way f'rom intinite separation. Such a system cannot be expected to yield very high densities, but can shed some light on the interactions whlch can occur. Figure 16 is a triangular plot of the experimental data. The general agreement with the treatment of Westman and Hug11l is very good. The agreement along the coarse-fine boundary is best, the coarse-medium next best, and the medium-fine the 
THE $\mathrm{UO}_{2}$ PARTICLE SYSTEM

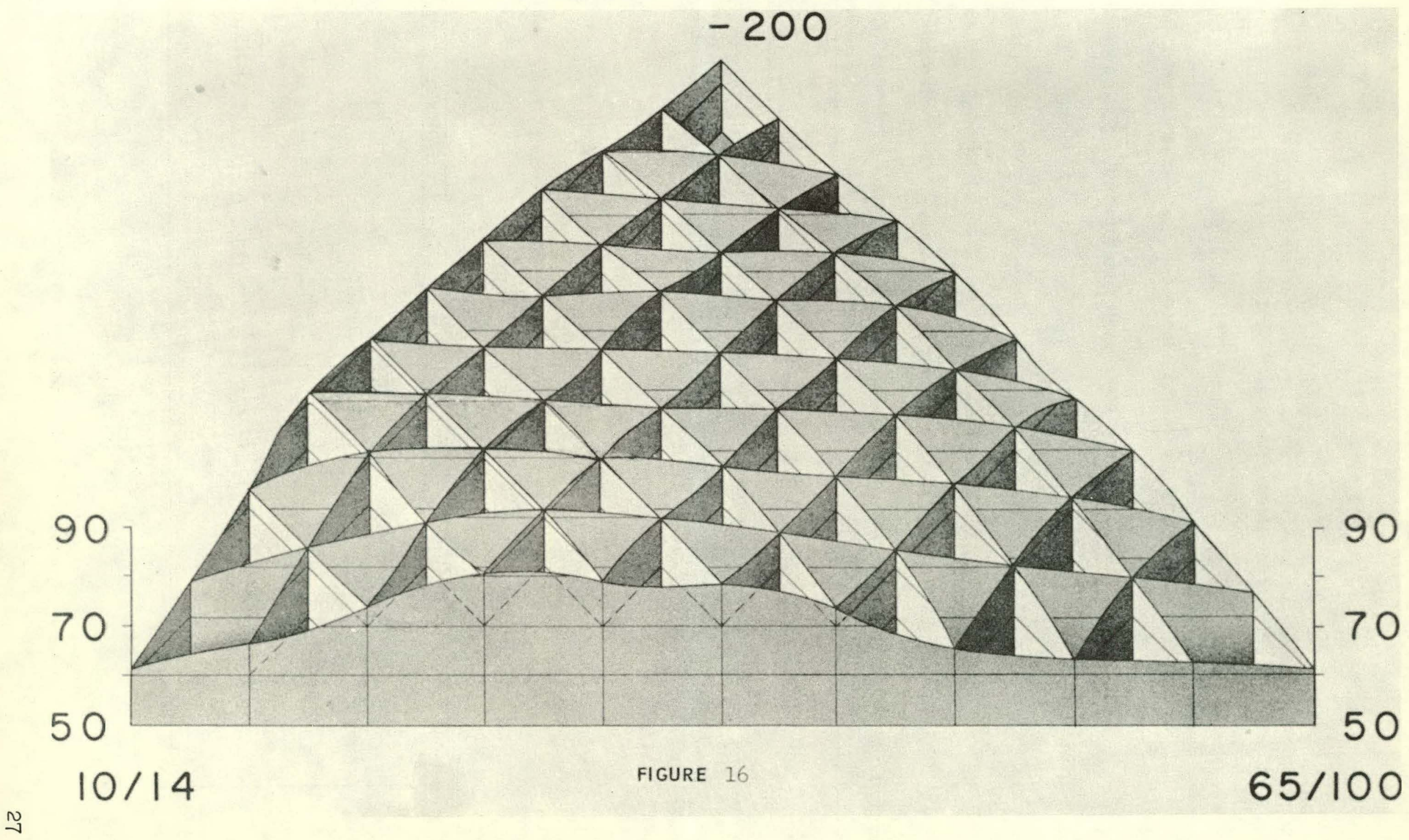


poorest, as would be predicted for the diameter ratios involved. From this diagram it seems evident that little hope can be held for attaining high densities outside of the region predicted by particle packing theory.

The best method of determining what the optimum composition should be for any three specific powder sizes in any specific geometry should be as follows: First determine the maximum of the coarse-medium binary. Second, draw a line from this hinary maximum composition un un equilateral t.riangle diagram to the fine corner. Third, determine the maximum of compositions along the line. With good luck, it should take about 8 to 12 experiments to decide on the optimum for that powder and that geometry. This point will shift with changes in powder character and geometry. Table III illustrates the sensitivity of density to composition. The composition would surely be different for a different powder and for other geometries.

\section{TABLE 1 II}

\section{SELECTED PARTICLE COMPOSITIONS}

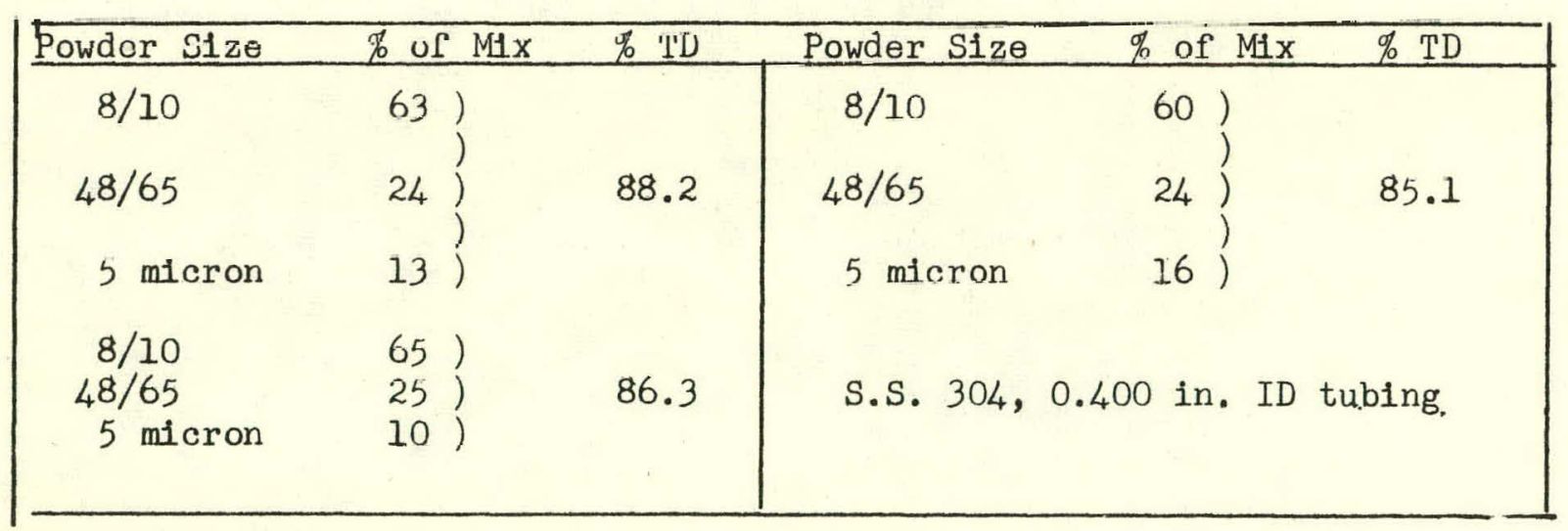


Table IV shows that shifting the middle fraction one size had no effect on the density, whereas shifting the coarse fraction down two sizes increased the density. These results point to the importance of precision fitting within a specific geometry.

\section{TABLE IV}

SELECTED PARTICLE DENSITIES IN 304 S.S. TUBING,

\subsection{0" DIAMETER}

\begin{tabular}{|lll|lll|}
\hline Powder Size & \% of Mix & $\%$ TD & Powder Size & $\%$ of Mix & \% TD \\
\hline $6 / 8$ & $60)$ & & $10 / 14$ & $60)$ & \\
$48 / 65$ & $20)$ & 86.8 & $48 / 65$ & $20)$ & 87.5 \\
5 micron & $20)$ & & 5 micron & $20)$ & \\
$6 / 8$ & $60)$ & & & & \\
$35 / 48$ & $20)$ & 86.8 & & & \\
5 micron & $20)$ & & & & \\
\hline
\end{tabular}




\section{$\checkmark$}

\section{THIS PAGE \\ WAS INTENTIONALLY \\ LEFT BLANK}


SECTION VI

\section{ARRANGEMENT OF POWDER SIZES}

It is apropos at this time to discuss the possible arrangement of powder sizes. The model which consists of particles of the coarse fraction touching each other, hexagonally closest packed on each layer, with cubic packing in a vertical direction as mentioned by McGeary and by Westman and Hugill, has already been discussed.

A new model is now proposed which will be called the emulsion model. In this model, maximum density is attained when each coarse particle is surrounded (and thus lubricated) by a layer of middles and/or fines. The maximum density is attaned at this composition because all powders are added simultaneously and this model leads to the least functional resistance. This model, therefore, calls for less coarse than the theoretical model calls for. There is evidence to indicate that perhups both models can and do obtain, and that roughly similar densities are observed. Combining both models would lead to a double-peaked phase diagram.

For hexagonal closest packing in any layer, the optimum size of particle would have a statistical diameter of $D / 3$ where $D$ is the ID of the tube. For a tube 0.400 in. ID, this would be 0.133 in. This diameter corresponds to about a 6 mesh size. If the particle were surrounded by a layer of $48 / 65$ at an average diameter of 0.010 in., then the basic coarse particle would only be 0.113 in. However, neither of these models fit Lhe data of Tableo III and IV. Insterad $8 / 10$ with $48 / 65$ fit the best. 
$8 / 10$ averages only $0.079 \mathrm{in.}$ which 1 s about $1 / 5$ of the diameter. When the shape of the particles is taken into account, and also the fact that the particles are not single sized but range from 1 to 1.4 in size, it can perhaps be seen that expecting the $D / 3$ model to fit exactly would be quite optimistic. However, by the time the model approaches $D / 5$ there is enough redundancy of fit to expect a reasonably good peak to occur. If perfect spheres of a known diameter were to be employed, the $D / 3$ model would porhapo be the bost iir.

After a great number of experiments on many kinds of powders, it seems reasonable to conclude that densities in excess of 8.5 per cent of theoretical are routinely achievable. Because of the great number of posaible formulas for achieving this goal, very excellent powder utilization can be expected, provided that at least 60 per cent of the starting powder is in the +20 mesh size range. Powders can be comminuted to achieve the necessary medium and fine oizes but there is no method, short of fusing, which can bulld up small particles into large particles.

Rods with starting densities of 85 per cent can be cold swaged to den. sities. of: the order of $91-92$ per cent with one nominal reduction not exceeding 40 per cent. Some internal clad indentation occurs with the larger particles but can be tolerated occasionally, especially in heavy-walled tubing.

There is nothing which precludes the possibility of achieving greater than 90 per cent TD by vibratory compaction alone with simple ternaries, especially if slightly broader bands are employed so as to obtain a little more redundancy of fit. The formulas so obtained, if any, would be unique to a given powder and geometry as well as to technique of fill and vibration. 32 
For general purposes, the size. ratios may approach 1:10:50 and still achieve greater than 85 per. cent. Spreading out the size ratios is beneficial; the one mix in Table III which went to 88.5 per cent was in the ratio $1: 48: 394$, which is perhaps excessive.

Wherever the ratio of any two sizes goes below about 1:7, particular attention must be pald to the blending beforehand to ensure adequate distribution since the particles cannot migrate through the holes. However, If the feed is slow enough, the effects of vibration are to open up the bed and allow migration and mass transfer.

Th1s problem w1ll be treated in more detall in the section on segregation, but it must be remembered that coarse fractions move up and small fractions move down. One should attempt to utilize this information by counter-current-flow techniques, making sure that the coarse fraction leads into the tube and the fine fraction enters last. One can ordinarily acti-, vate about $2 \mathrm{ln}$. of powder helght in a small-dlameter tube. Hence, each 2 in. equivalent ought to contain all the ingredients needed for the correct density. 
THIS PAGE

\section{WAS INTENTIONALLY LEFT BLANK}


SECTION VII

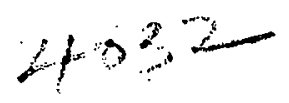

\section{QUATERNARY AND HIGHER SYSTEMS}

From an analysis of many ternary systems it soon became apparent that densities in excess of about 87 per cent of theoretical would be obtained either by very severe working of ternary mixes or by using more than three particle sizes to begin with. Only limited work has been done at this time on the higher systems but that which has been done is encouraging. All mixes which achieved more than 88 per cent have been more than 3 sizes. One mult1-component mixture has been brought to 92.8 per cent. The approach to the quaternary system has been by the cascade binary route in some instances, and in others by a strictly emplrical approach. This study has not been completed at this time and it would be difficult to make a definflive statement about achleving greater than 90 per cent routinely. Several false starts have been made and the best or optimum approach is still open. One of the first approaches was to try a cascade binary using immediate-nextdoor neighboro. Sinco moot binary maxima occur in the region of 70 per cent coarse and 30 per cent smaller, a matrix of $35 / 48$ at 30 per cent was used in the system 6/8, 8/10, 10/14. Figures 17, 18, 19 summarize the data taken here.

of particular interest is the undulating nature of the density-composition plots. These data are highly reproducible and the variations are real. Each point is good to at least \pm 0.1 per cent at the level indicated, i.e. $74 \pm 0.1$ per cent. Notice that the curves with $35 / 48$ added 

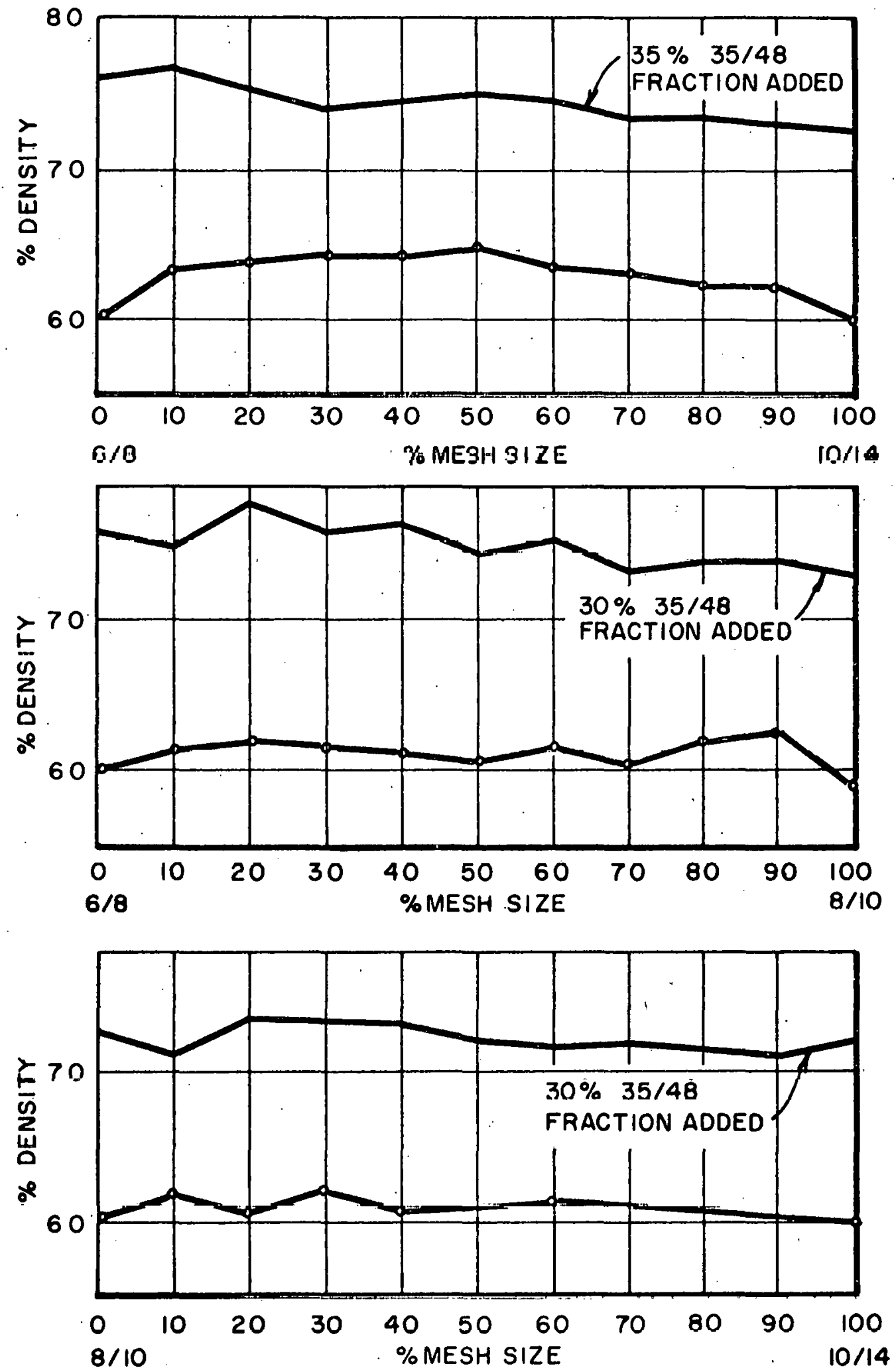

FIGURES 17 (TOP), 18 (MIDDLE), AND 19 (BOTTOM) BINARY DIAGRAMS OF TERNARY SYSTEM

$$
6 / 8-8 / 10-10 / 14
$$


are more grossly periodic than the simple binaries themselves. This is interpreted as resulting from the adding and subtracting action of the small $35 / 48$ onto the larger $6 / 8,8 / 10$, or $10 / 14$. The high redundancy of fit whlch is possible when more than one size is used 1s again illustrated.

All of the systems exhibit maxima and minima, but none of the minima go below the density of the lowest member. It is to be expected that the minimum, in some systems, could go below the density of the lowest member. The data are even more interesting when replotted on triangular paper. Figures 20, 21, 22 illustrate these data. It can be seen that in all cases the maximum in the ternary system lay on the line joining the coarsecoarse binary maximum to the $35 / 48$ corner. In one case, namely $6 / 8-10 / 14$, $35 / 48$ the most maximum point lay off the line. There is reason to belleve from later work, as will be shown, that there is a high probability that a maximum would be found at the point where the line from $35 / 48$ through the 77 per cent value intersected the 6/8-10/14 edge.

Some systeins show a sudden rise and fall at very critical fit positions where the particles either just fit or are badly out of fit. It is possible that the true maxlmum was missed in that ono inotance.

From the work on the system $6 / 8,8 / 10,10 / 14$, a composition which yielded 66 per cent TD when vibrated was chosen for use as the coarse phase in the following:

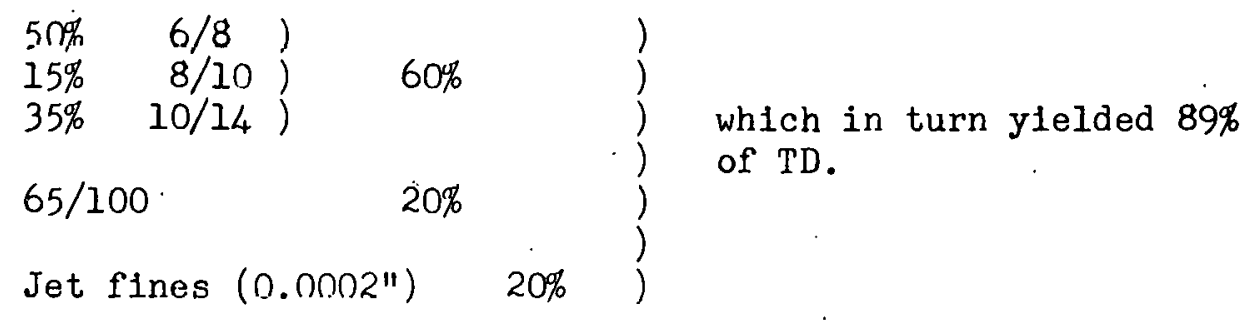




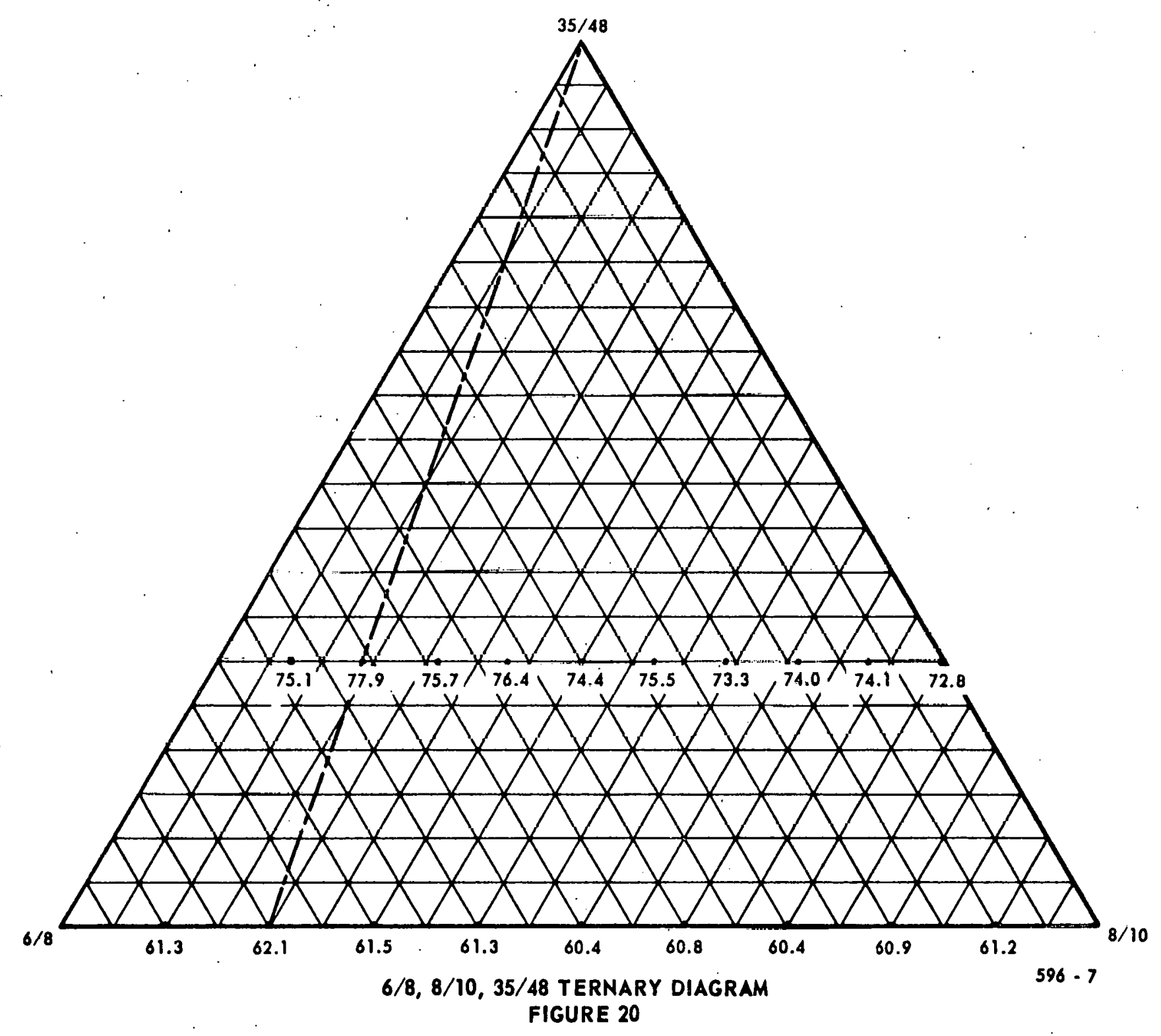




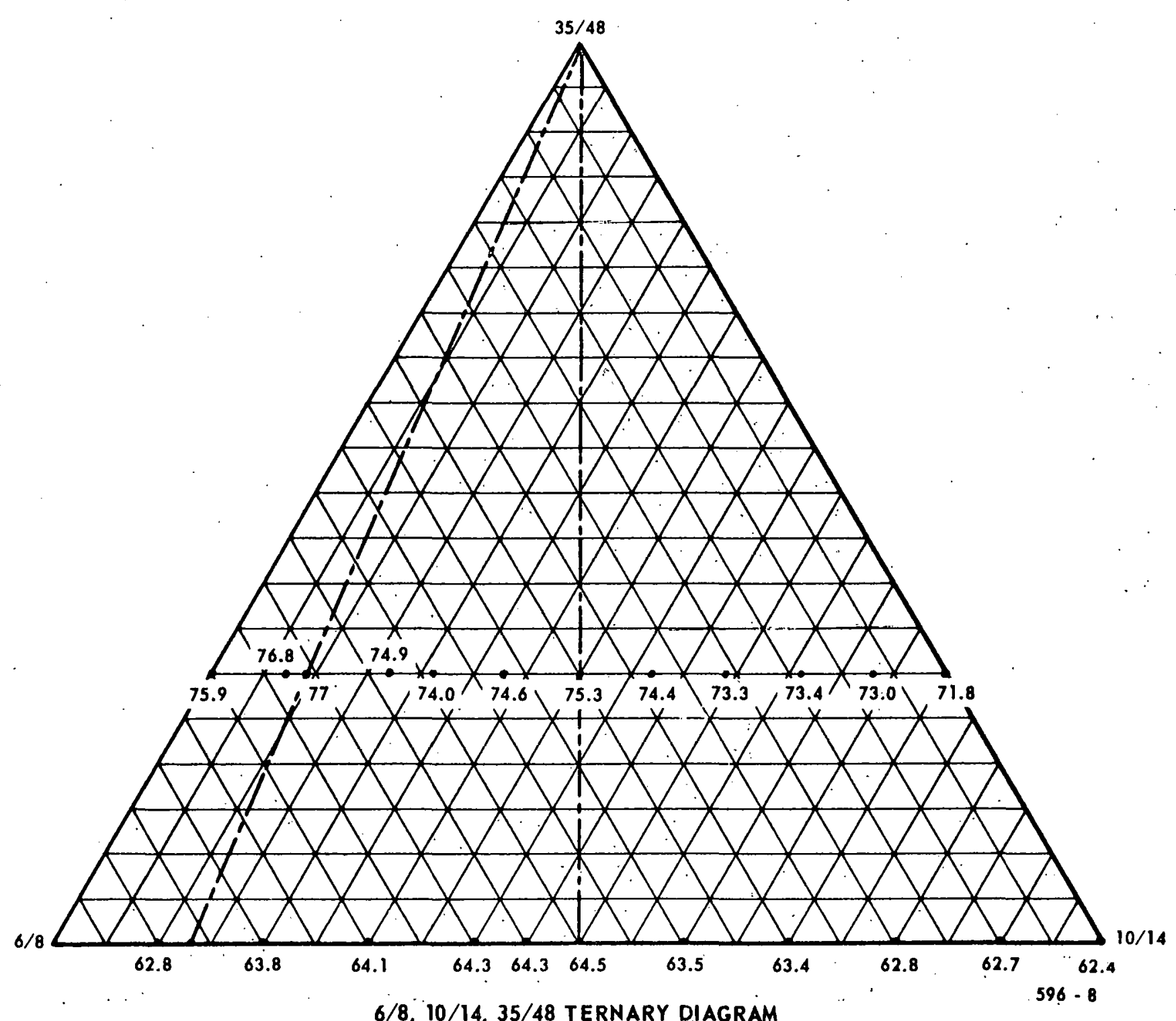
$6 / 8,10 / 14,35 / 48$ TERNARY DIAGRAM FIGURE 21 


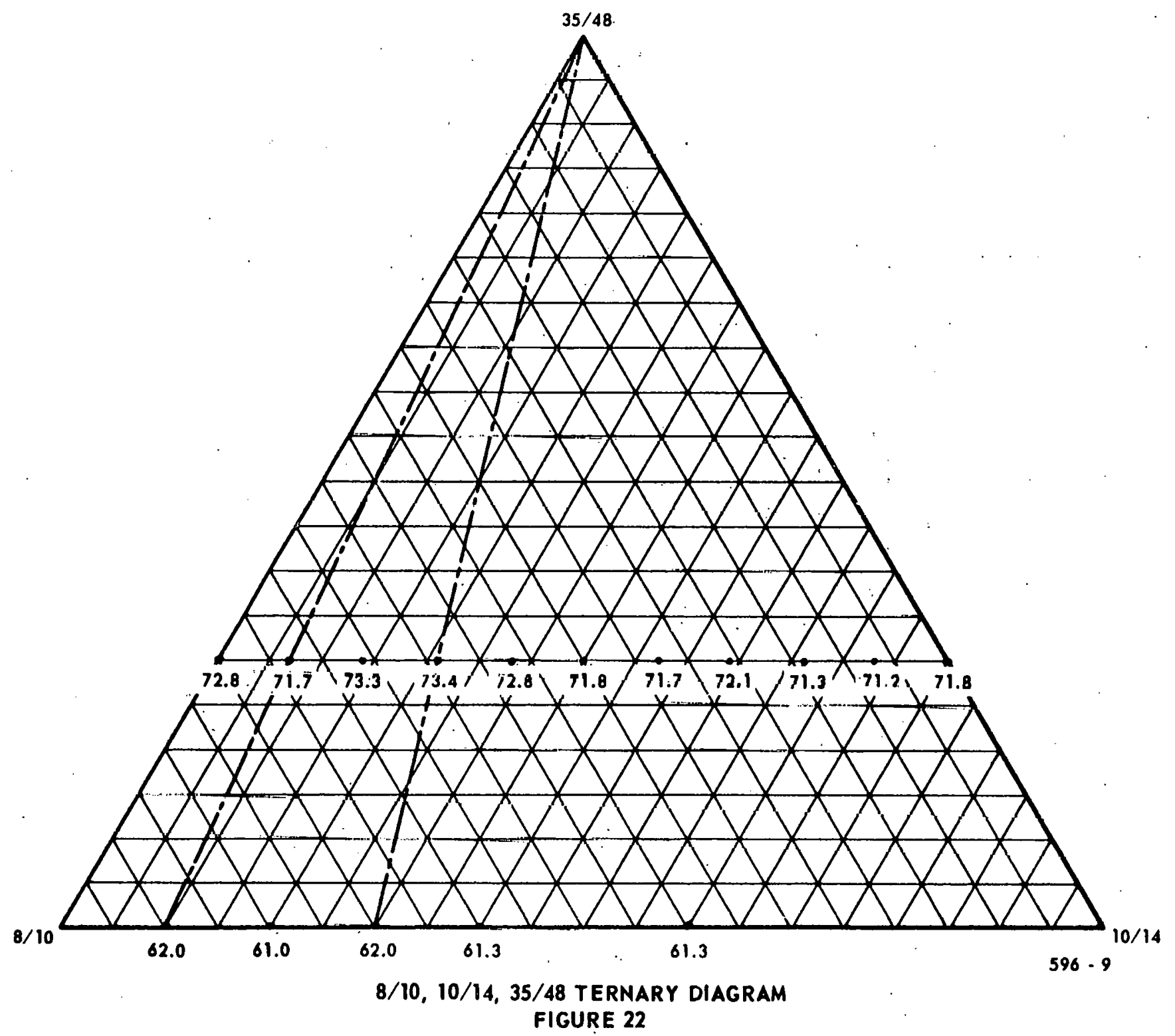


A simple binary mixture of this same coarse and jet fines in the proportion 65 per cent coarse and 35 per cent fines yielded 87.8 per cent TD.

It would seem that the surface has hardly been scratched at this time, and surely continued exploration will open still further pathways. 
THIS PAGE

\section{WAS INTENTIONALLY LEFT BLANK}


SECTION VIII.

\section{SEGREGATION}

It is a well known fact that under vibration a mixture of particles will separate, with the heavier and/or larger particles rising above the lighter and/or smaller particles. This relative movement is related to Newton's laws of motion, and to the laws of the conservation of momentum. If particles are accelerated in a given direction by the shaker table and then the accelerating force is removed by the reversal of the table, then for a given final velocity of the shaker table and tube containing the particles ä larger mass posisesses greater momentum and hence against the retarding force of gravity will move a greater distance before the kinetic' energy is dissipated by being converted into potential energy. Even if the larger particles are of lower density (and thus posibly lighter than the smaller particles), the smaller particles will work into the interstices between the larger particles; segregation is achleved in the same $:$. manner as. is seen when rocks rise to the surface of the ground. As imight be suspected, certain frequencies, related to the size and mass of the particles, are more efficient at performing this segregation than others. The greater the linear displacement of the table and the greater the force, the more raplaly the segregation occurs.

In particle packing in tubes, the lower frequencies are most effective, with frequencies of the order of $250 \mathrm{cps}$ being quite effective for most systoms. To prevent segregation, the experimenter has at least two 
possible routes to follow. The first and' most obvious is to put a sufficient restraining force on the top of the column to prevent individual particles from leaving the upper surface and moving in free air, but still of small enough magnitude that some degree of freedom of rotation and small translation is permitted to aid in migrating particles to the position of lower energy. This is very important when working with single frequencies, at high G levels and large displacements. When the displacement is held

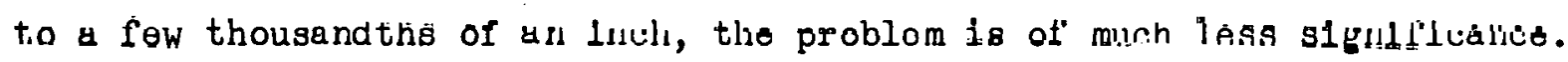

The other, not-co-obvinus snlulion has been used with suncess - the use of a combination of frequencies approaching a nuise spectrum. Thus, high $G$ levels can be obtained with small displacements and enough randomness of motion to forestali any aggressive segregation. However, even with the noise spectrum, segregation can and will occur if vibration is cont.inued at length. The use of noise, or mixed frequencies, has been especially helpful in the practical solution of the loading problem.

During the initial filling of the tube it is desirable to achieve high densities by aggressive vibration before a long column of powder increases the frictional forces and delays the attainment of high density. If pure sine waves are used, then very low $G$ forces must be employed. By using noise, high G levels can be used with consequent excellent packling.

After the tubo is loaded, a restraining force can be applied and high G level pure sine waves of varying frequency can be employed to effect a considerable improvement in density.

When systems were studied in glass and plastic tubes, segregation could be easily followed. If pure sine waves were employed with no restraint 
and at high $G$ forces, ternary blends could be cleanly separated in three distinct bands: coarse at the top, middles in the middle, and fines at the bottom. Quaternary mixes were not studied. 


\section{THIS PAGE \\ WAS INTENTIONALLY \\ LEFT BLANK}




\section{POWDER UTILIZATION}

It has been shown that if the fines one is using will pack to densities of the order of 60-plus per cent, this same density can be achieved by packing in the volds between the larger particles, provided that the difference in size is of the order of 20. Thus, if densities of 87-90 per cent are desired, the cut-off density for mixtures containing part1cles 20 times larger than the fines must be around 73-75 per cent. For example, suppose some fines capable of packing to 60 per cent alone are avallable and their average size is 0.0002 in. diameter. Then the average size of the next higher particles should be not less than 0.004 in., or about $100 / 150$ on the Tyler scale. Table V demonstrates what might be achieved if various coarse-medium densities are achieved.

\section{TABLE V}

\section{FINAL DENSITIES PREDICTED FOR VARIOUS COARSE DENSITIES}

$$
\text { (Density of Fines at } 60 \text { Per cent) }
$$

(1)

\begin{tabular}{|c|c|c|c|}
\hline $\begin{array}{c}\text { pensity Attained } \\
\text { by Sizes } \\
\text { Above 100/150 }\end{array}$ & $\begin{array}{c}\text { Volume Remaining } \\
\text { To Be Filled }\end{array}$ & $\begin{array}{c}\text { Contribution } \\
\text { of Fines } \\
60 \% \text { of (2) }\end{array}$ & $\begin{array}{c}\text { Final Density } \\
\text { Sum of (1) and (3) }\end{array}$ \\
\hline 90 & 10 & 6 & 96 \\
85 & 15 & 9 & 94 \\
80 & 20 & 12 & 92 \\
75 & 25 & 15 & 90 \\
70 & 30 & 18 & 88 \\
65 & 35 & 21 & 86 \\
60 & 40 & 24 & 84 \\
55 & 45 & 27 & 82 \\
50 & 50 & 30 & 80 \\
\hline
\end{tabular}


In actual practice it is observed that the coarse-medium densities lie in the region of 60 to 75 per cent, thus guaranteeing achievement of at least 84 per cent to 90 per cent TD. It appears possible to achieve 72 per cent with many combinations of sizes larger than 150 mesh. This happens in binary, ternary, and higher systems. A system of linear programming will be required for utilization of powder in an efficient manner. Following is an outline of a suggested procedure. The material is assumed to have been ordered as a $6 / 20$ mesh arc-fused powder.

1. The powder 1e etarted through the aleving Sweoo or equivalent sys.tem.

2. After one or two batches have been sieved, and a sieved powder inventory is on hand, trial runs can be started.

3. Material in the -20 range is ground to move powder into the $30 / 60$ range.

4. -60 mesh material is sent to the jet mill for creation of jet fines.

5. Each of the upper size ranges are tried for binary maxima with middles, say $30 / 60$.

6. Those which can make 73 per cent are used with fines.

7. Tho romaindor must bo oombinod to reaoh the goal.

A representative system is as follows $\left(\begin{array}{l}6 / 20 \\ \text { wag ordered }\end{array}\right)$ :
$6 / 8$
0
$8 / 12$
$\sim 19 \%$
$12 / 16$
$32 \%$ 


$\begin{array}{ll}16 / 20 & 25 \% \\ -20 & 24 \%\end{array}$

The first formula will be 40 per cent $8 / 12,30$ per cent $16 / 20$, 30 per cent. $30 / 60$, plus fines to make target goal. Portions of $12 / 16$ will be ground to $30 / 60$ range and to jet fines. Middles and fines are required to the extent of about 40 per cent. Thus at least 16 per cent must be obtained from higher fractions. The f'irst formula will consume all of the $8 / 12$, 14 per cent of the 16/20, leaving 11 per cent $16 / 20$ on hand. The 11 per cent $16 / 20$ will be added to the minus 20 mesh to make 35 per cent -20 . Now trial runs will be made on $12 / 16$ with $30 / 60$. The target of 73 per cent is reached at 70 per cent 12/16,30 per cent 30/60. Fortunately, the yleld of ground powder in the desired range is a little over 50 per cent. Thus it is possible to grind and not accumulate too many fines.

Toward the end of the run, it may be that a third formula must be sought which will achieve the same target density. In all cases, care must be taken that powder is not moved down to the fine sizes so rapidly that material which can only be used as jet fines does not accumulate much above 15 per cent of the total, until it is certain that more will be required. Ordinarily, this is no real problem, but sizes to be ground must be selected with care. It is better to keep other middle fractions on hand so that other ternary mixes can be used to achieve the desired goal.

In the case of some rods made for a VBWR assembly, a simple serialdegradation of sizes was used, with a very limited amount of powder, to achieve greater than 85 per cent in all rods. The mix started out as 
$6 / 8,35 / 48$, and fines in $60,20,20$ proportions. When the $35 / 48$ was consumed, the formula was switched to $48 / 65$. After the $6 / 8$ was all gone, it switched to $8 / 10$, and finally to a mixture of $8 / 10$ and $10 / 14$ and $65 / 100$. In all instances, target densities in the 87 per cent range were obtained. Better results might have been achieved if the mixes had been optimized for each size on hand. However, for this particular application it was not necessary

It romalno to bo demonstrated on a large l'ui of sevel'ul hurdred or thousand segments as to the exact nature of the 1.1near programming difficulties to be encountered. It should be evident that a successful powder line w11l incorporate methods of grinding to the $-20+150$ range and also a way of comminuting powders to the 1-10 micron range. 
SECTION X

\section{LOADING AND FILLING TECHNTQUE}

Many different loading and filling techniques have been employed but the presently-preferred method is the one described below. For the development phase of the work, all tubing used was calibrated for volume by filling the tube with water or alcohol from a buret and calculating the volume-per-inch from the length measuremont. After a tube had been filled with a welghed quantity of powder, a flat-footed probe was pressed on the upper surface of the powder and the position of the top edge of the tube to a referenco mark was measured with a ruler. For conventence purposes a table was prepared for a 500-gram sample of powder in a particular tube, giving the density directly from the measured distance.

For other weights or other tubes, the formula uoed was a follows: Per cent of $T D=\frac{\text { (weight of powder) }(100)}{\text { (tube length-depth to interface) (10.97) }}$ (factor) where the factor ss the cubic centimeters per linear inch. All lengths are in inches and weights are in grams. Thus, only an over-all or effectIve density was calculated in every instance. It is obvious that regions of inhomogeneity could exist, but by successive sampling techniques it was soon learned that high density and homogeneity go hand-1n-hand. Reference to a coarse-fine binary is probably the best way of demonstrating the pitfalls involved. 
Assume a matrix of coarse particles within a tube at a fixed height. This height is held by a restraining member throughout the experiment. Let the mass of the coarse be $m_{c}$ and the volume of tubing defined by the height be $V$. Then the starting density is $\frac{m_{c}}{V}$. If fine powder is now fed in, it will migrate to the bottom of the matrix and pack in the holes there to some maximum local density. The over-all density will now be $\frac{m_{c}+m_{F}}{v}$, but obviously only the lower portion is really of greater donolty. Not until the binary maximum is roaohod whoro tho fino powdor just equals the height of coarse matrix will the density be correctly calculated. Thus, it is almost imperative that only mixes in the region of maximum be used when uniform density is desjred. To some extent the same reasoning will hold for multiparticle mixes, but the region of homogenelty is quite a bit broader in the practical application where the whole $\mathrm{mix}$ is fed into a tube at one time. If the $\mathrm{mix}$ were fed in successively, then the same reasoning could be expected to hold.

\subsection{Preliminary Blending}

This brings up the question of blending prior to a run. If the mix has been made up in proportions exceeding $1: 7: 49$, then blending is not nearly as necessary as in other mixes, but it is imperative that measures be taken to insure that the first particles entering the tube are the coarse particles. When the size ratios are as given or even more, the middles can migrate through the coarse, and the fines can migrate through the. coarse and through the middles with the aid of vibration at least for short distances. It again will be stressed that coarse particles must be the first to enter since there is no way, short of turning the tube over, of causing the coarse particles to migrate through middles or 
fines to achieve uniformity in the bottom portion. As the proportions go down from the 1:7:49 values, it becomes much more important to blend thoroughly before filling, and it becomes even more essential to insure a uniform feed to the tube.

It is actually surprising to see how uniformly the tube can be. packed despite all the theoretical reasoning which would tend to convince one that segregation would be a problem. It should be remembered that vibration causes the lattice to loosen up and hence permit some migration of particles from one point to another. Again, it is desirable that the largest particles enter the tubing first. When a Syntron feeder is used, this tends to happen automatically, because the large particies teind to lead down the tray.

In some instances, mixes have been made up by weighing first the fines, then the middles, ard then the coarse into a jar. The jar was Inverted into the cup of the Syntron feeder and fed to the tube without blending. When the proportions of size were close to $1: 7: 49$ or greater, the same density was achieved as in well-blended samples. Maximum density tonok snmewhat longer to attain, by ? to 3 minutes. Even mixes in lower proportions have been successfully loaded by this technique.

Occasionally a certain $\mathrm{mix}$ whlch has unfformly been yielding a certain density time after time with maybe \pm 0.1 or \pm 0.2 per cent variation, will all of a sudden be 5 per cent low or so. These are called "statistical goofs", and are usually caused by fine powder leading into the tube, and other times by bridging of some very irregular particles. In essentially all instances, the powder can be unloaded from the tube, 
the tube refilled with the same powder, and immediately attain the right density. Thus a certain amount of art is involved in loading tubes to uniform density.

However, in order to achieve a certain density the primary factor is choice of $\operatorname{mix}$ and the secondery factor is art or technique. It would be interesting to set up an experiment where the coarse was fed from one fovder, the medium from another, and the finos from another with the speeds so adjusted that coarse led in first, middle second and fines last, with the order remaining throughout the feed period.

\subsection{Filling Procedure}

After the method of feeding has been mastered, the actual filling will start. The five oscillators have been set to a set of frequencies which initially were selected on the basis of system resonances. However, later work showed that this was not a necessary criterion and that a well-blended noise was all that was needed. The wave shupe can be seen by observing the output on the oscilloscope and can be felt by lightly fingering the tubes. A good setting will have a slightly undulating pattorn on the oscilloscope; the pienes of tuhtng will feel very mach alive. More than anything else this feeling of "aliveness" is a good indication, and an operator soon learns that the tubing is activated over its entire. length and not just at certain spots. The oscillator settings are not critical but the following is a good guide.

The first oscillator is set oi some resonance in the vicinity of $1000 \mathrm{cps}$, being careful not to chouse a table or case resonance which may be present there. Number two is set between 500-600 cps (where powder 
is activated), but avoiding a sub-harmonic of number one oscillator. Number three is set on about $250 \mathrm{cps}$ : Number four should be set at about 90-100 cps, and number five is used to "fill in" the scope picture to smooth the pattern out and avoid beat phenomena. One favorite setting 1s as follows:

$$
\begin{aligned}
& 1=960 \mathrm{cps} \\
& 2=600 \mathrm{cps} \\
& 3=500 \mathrm{cps} \\
& 4=250 \mathrm{cps} \\
& 5=92 \mathrm{cps}
\end{aligned}
$$

Thls setting has been used for much of the work. It is blended with different amplitudes to produce the effect desired.

After the oscillator settings have been made and the particular tubes are very much alive and the subjective feeling of good settings has been made, actual loading will start. Despite the fact that the noise w1ll minimize segregation, the first portion, about three to aix inches, 1s always loaded with G forces around 5-7 G's. As the loading progresses the amplitude control is gradually advanced so that the maximum drivercoil current is being used by the time the colurn is around two feet hlgh. Vibration 1o stopped immediately upon completion of feeding. The restraining foot is lowered into place and a slight pressure ( 1-2 psi) is applied. The vibration is started again and brought up to maximum G level. The vibration is stopped about every minute to see if further gains are belng made. When maximum density has been achieved, the nolse 1s switched off and a pure sine wave is applied, starting with 1000 cps and sweeplng down to 200 cps at the rate of $31 \%$ minute on the $M B$ oscillator. 
The sweep goes back to 1000, down to 200, back to 1000, and is shut off. Ordinarily this is the end of the run, but occasionally some further, very slight, gain can be had by switching back to the noise again, and then back to a sweep.

During the first sweep there will be certain frequency regions where the marker foot will show a sudden drop indicating a densification has occurred. It does no good to stop at this point and continna furthar vibration at this frequency. 'I'he ef'fect is seen only while the frequency 1s changing and is most pronounced at the speed of sweep mentioned. Apparently the system must move into and out of resonance in order for this to occur.

The most difficult kind of rod to make is the kind that calls for powder to within a quarter of an inch or so from the top edge of the tube. In order to fill these, the rubber funnels were designed with a straight length of about two inches before flaring out to match the feeder. Excess powder over that which goes in during the fill cycle remains in this region. When the foot is placed on the powder there is no clearance around the foot, and as the sweep proceeds the powder will work down into the tubing.

Whether the tube requires a certain interface depth or a certiain density makes little dit'terence. In general, a fixed weight of powder is put into a tube rather than a certain density. Since commercial tubing ordinarily varies a little in diameter, either the interface or the density must vary for a fixed weight of powder. Since the reactor designer is most concerned with the core load, the weight is held constant. 
After filling, a temporary plug or cork is inserted into the end to minimize contamination spread when the tube is removed. The end which was in the box is wiped clean and the rod sent to a welding station. It has been found very beneficial to have all vibration controls and air pressure controls remotely located from the console, such that the operator has complete control of the situation at all times.

After many runs it was noticed that there was a tendency for a little powder to remain in the Syntron at the end of a moderate feed. To overcome th1s, a Variac has been inserted in series in the line. It is set low for the feed and then at the end, the operator increases the line voltage to all feeders simultaneously. This vigorously vibrates all Syntrons and insures a complete delivery of even the finest powder. These latter techniques are of most use in production, since a highly flexible arrangement is desired. Each Syntron can be individually set by its own control to account for dif'terences in behavior. Also, all can be raised or lowered in amplitude simultaneously to adjust the gross feed rate. 
THIS PAGE

\section{WAS INTENTIONALLY LEFT BLANK}




\section{SECTION XI}

\section{POWDER WORKABILITY OR FRIABILITY}

It has been frequently observed that substantial changes in powder. size composition occur during vibrational compaction. This ability of the powder to break down, round off corners, and grind into the available hole may be directly related to the abillty to go to high dens1ties. It probably accounts for many of the discrepancles observed around the country where the use of identical starting formula's do not lead to the same final density. All of the data have been reported in the form of starting formulas and very little in the form of final composition. This is somewhat due to the fact that postvibrational sieve analyses are laborious, tedious, and time-consumIng.

An attempt was made here to evaluate in a semi-emplrical way the differences in powder character, in such a manner as to pinpoint the degree of breaking-up which had occurred. It was felt that arc-fused powder, having cooled in some extremely steep thermal gradients, would be highly stressed. The relief of stress during vibration would account for a large measure of the attrition. Annealing the powder in hydrogen at temperatures well into the plastic range of $\mathrm{UO}_{2}$ should aid in relieving the stress and make for a much tougher ceramic.

\subsection{Experimerital Procedure and Results}

The procedure chosen was as follows: 
1. A 7/16 in. OD, 0.020 in. wall stainless steel tube, 46 in. long, with a stud-type end plug was used.

2. The oscillators were set as follows: .
a. at $30 \mathrm{cps}$ to $10 \mathrm{G}$ level
b. add $95 \mathrm{cps}$ to $20 \mathrm{G}$ level
c. add $250 \mathrm{cps}$ to $30 \mathrm{G}$ level
d. add $1000 \mathrm{cps}$ to $40 \mathrm{G}$ level:

3. A total of 200 grams of trial powder was used in the followIng proportions:

$6 / 8 \quad 160$ grams . $80 \%$

$8 / 35 \quad 0 \quad: \quad 0$

$35 / 42 \quad 40$ grams $20 \%$

$-42000$

4. The powder was poured all-at-once into the tube without v1bration.

5. The depth to the interface was measured.

6. Power to $40 \mathrm{G}$, vibrated exactly 30 seconds.

7. Measured depth.

8. Repeated for total of 2 minutes.

9. Measured interface depth each time.

10. Performed a sieve analysj.s as

$$
\begin{aligned}
& +8 \\
& 8 / 35 \\
& 35 / 42 \\
& -42
\end{aligned}
$$




\section{TABLE VI}

ATTRITION OF POWDERS DURING VIBRATIOL:

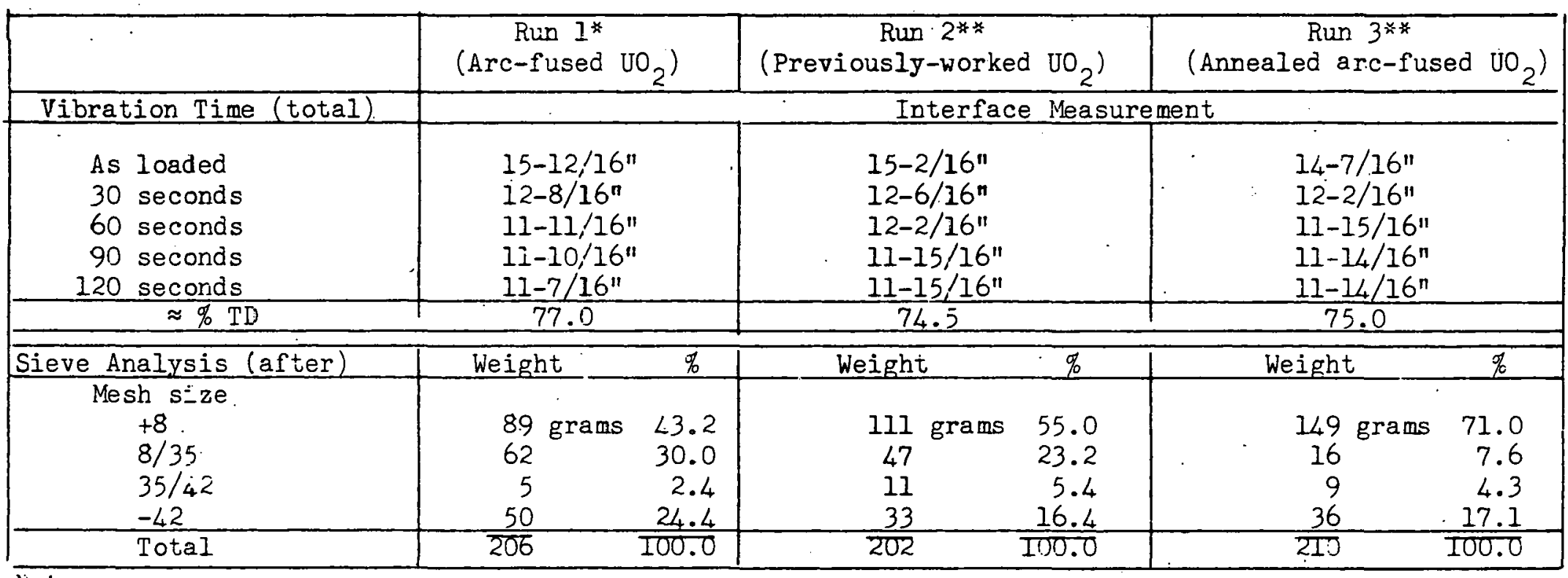

Notes:

*Notice the very great loss of the $35 / 42$ fraction because it was crushed between particles of the $6 / 8$ mesh. The $6 / 8$ mesh also lost almost half, and notice that it moved down to -42 mesh, not just to the next bracket.

* The powder used had already been ased in several experimental runs; that is, it had already been worked $=0 \cdot$ some extent: The $6 / 8$ mesh fraction moved slightly into the $8 / 35$ range, and the $35 / 42$ to the -42 range. The lesser wors also resulted in a decrease of about 2.5 per cent final density. Whether these results are reproducible is not known at this time.

**24 pounds of arc-fused majerial was annealed in the regular sintering furnace for about 12 hours (a) $1650 \mathrm{C}$. The aggregates were broken up and the work run made. Again, notice that annealed powder did not change as much as green powder did. Also, notice the difference of about 2 per cent final density for supposedly similar mixes. 


\subsection{Evaluation of Experiment}

Not much further work was done on this particular idea, but the work which was done did serve to illustrate the possibility of some differences between powders, which might explain some of the observed data. Rather than trying to characterize all powders; it was felt that a more profitable approach would be to find the optimum density for each new batch of powder in the system of interest.

At this lime $1 \mathrm{l}$ inlghl be hypolhesized lhal a powder's breaking up and relleving the stresses, at the same time causing (or enabling) the particles to fit into the holes avallable for them, is a necessary procedure for achleving maximum density with angular, odd-shaped particles. This may not be true with spherical particles wnich have been chosen in the right size relationships.

The shift in density noted for the 80-20 per cent binary under test may mean that a shift to another composition would yield the same final density. At this time it would be hypothesized that more fines and less coarse would achleve the same results with highly-worked or stress-relieved powder, as with green powder.

During these "work" runs and also during the ordinary runs, significant hot regions can be felt on the tube walls as the vibration proceeds. These hot spots are undoubtedly caused by the mechanical heating caused by stress relief during vibration. The lack of this tube heating has been intuitively associated with low density runs, although no definitive experiments have been run which would demonstrate such a correlation. In some instances the tube wall gets so hot that 
it is impossible to hold the hand on the tube more than a second or two. There is some concern that with fine powders such heating could lead to oxidation of the uranium dioxide, but such oxidation has not been observed as yet. It is estimated that temperatures around 60-65 C have been occasionally produced, but they are the exception. Temperatures around $30-40 \mathrm{C}$ are more common. 
THIS PAGE

\section{WAS INTENTIONALLY LEFT BLANK}




\section{RECOMMENDATIONS FOR FURTHER WORK}

Additional work on the cascade binary system would undoubtedly be valuable, from the standpoint of yielding controlled, higher densities.

Another area of profitable work would be in the selection of 100 per cent-dense larger particles and medium particles in order to assess the value of high particle density.

The study of the fines, as mentioned above, will proceed at a slow rate under other funds and will undoubtedly yield valuable information.

Finally, a rigorous investigation of the effect of powder friability upon final density would be in order. This one area probably contains the key to the variable results of the many investigators. 
APPENDIX

FORMULAS FOR DENSITIES ABOVE $75 \%$ TD

(A figure in parentheses indicates a brosd fraction with limita indicated by positione of the brackets.)

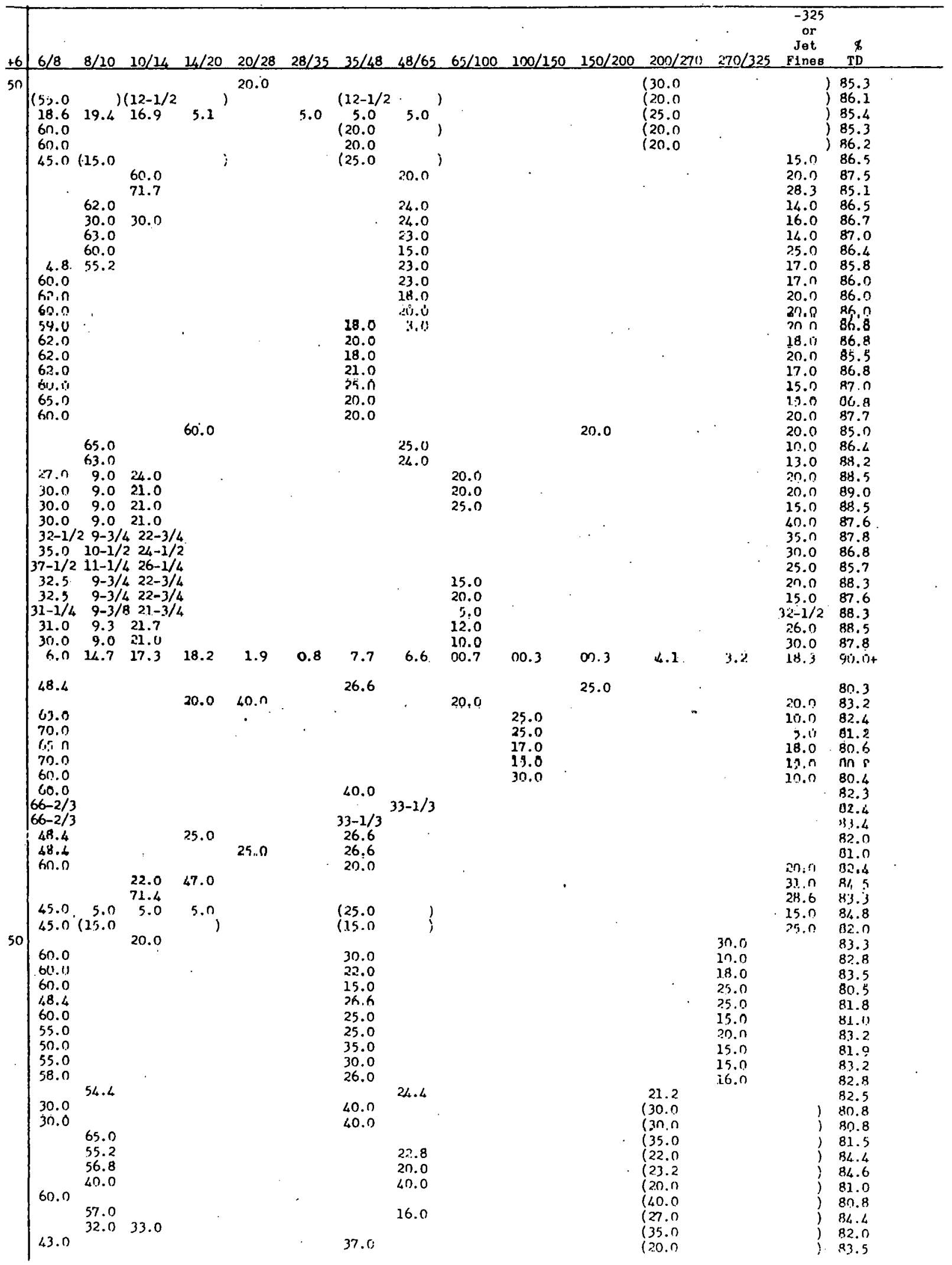




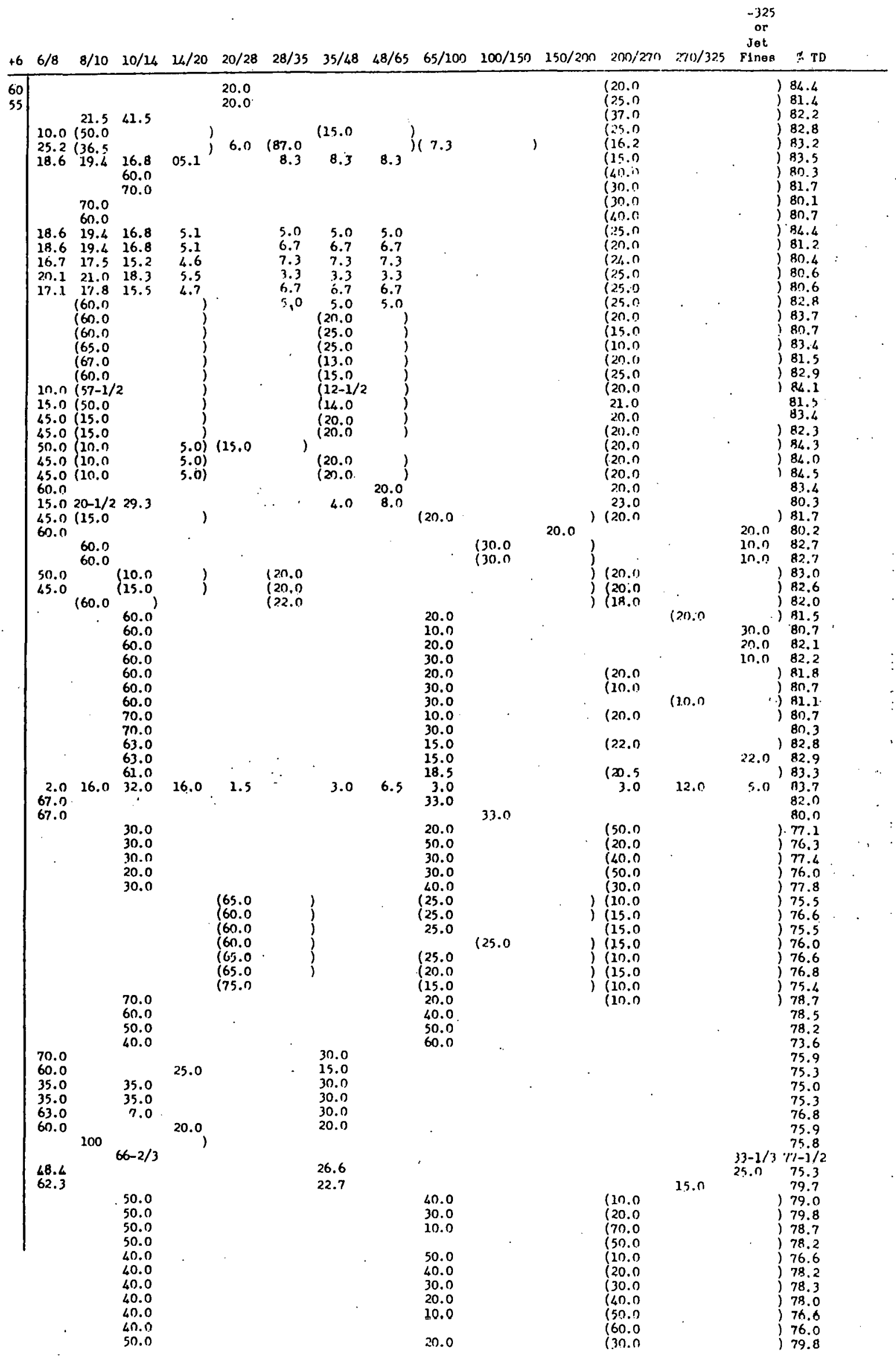




\begin{tabular}{|c|c|c|c|c|c|c|c|c|c|c|c|c|c|c|}
\hline $16 \quad 0, / 8$ & $8 / 10$ & $10 / 14$ & $14 / 20$ & $20 / 28$ & $28 / 35$ & $35 / 48$ & $48 / 65 \quad 65 / 100)$ & $100 / 1.50$ & $-150 / 200$ & $0 \quad 200 / 270$ & $2 m / 325$ & $\begin{array}{c}-325 \\
\text { or } \\
\text { Jet } \\
\text { Fine }\end{array}$ & \&TD & \\
\hline & & 30.17 & & & & & 50.0 & & & $(20.0$ & & 1 & 76.3 & \\
\hline & & $3 n .0$ & & - & & & 30.0 & & & 140.0 & & 1 & 77.3 & \\
\hline & 50.0 & & & & & & & & & 150.0 & & $i$ & 77.8 & \\
\hline & & & 20.0 & & & & 40.0. & & & $(4 \cap 0.0$ & & ) & $7 f, 2$ & \\
\hline & & & 0.0 & & & & 50.0 & & & (30.0 & & 1 & 76.5 & \\
\hline & & & & $(6,1) .0$ & 1 & & (2). $n$ & & & )$(20.0$ & & $\therefore$ & 75.9 & \\
\hline 20.0 & 21.0 & 1 म. 3 & 5.5 & & $3.0^{3}$ & 5.0 & 5.10 & & & $(20.0$ & & j & 78.2 & \\
\hline 15.5 & $16 .:$ & $1 / 4 . i$ & .1 .25 & & 3.3 & 8.3 & a. & & & $(2 ;) 0$ & & 3 & 78.2 & \\
\hline 17.0 & 17.8 & 15.5 & 4.7 & & 8.3 & X.3 & 8.3 & & & $2 n . n$ & & ; & $79 . \%$ & \\
\hline 17.0 & 17.8 & 15.5 & 4.7 & & 10.0 & 10.0 & 10.0 & & & (15.0) & & ? & 79.6 & \\
\hline 18.6 & 19.4 & 16.9 & 5.1 & & 3.3 & 3.3 & 3.3 & & & 30.0 & & ) & 76.9 & \\
\hline 10.6 & 10.8 & 9.4 & 2.8 & & 11.0 & 11.0 & 11.0 & & & $(33-1 / 3$ & & ) & 77.8 & \\
\hline 45.0 & (15.0 & & & & & $(20.0$ & ) & & & $(20.0$ & & ? & 78.7 & \\
\hline in. 0 & $(60.0$ & & & & & 110.0 & i & & & $(20.0$ & & ) & 77.6 & . \\
\hline 50.0 & 7.0 & 11.0 & 14.0 & 3.0 & & & & & & (15.0 & & j & 76.6 & \\
\hline 50.0 & 10.0 & & 10.0 & 5.0 & & & & & & $(30.0$ & & ) & 75.8 & \\
\hline 50.0 & 5.0 & & 10.0 & 5.0 & & & : & & & (30.0 & & ; & 78.7 & \\
\hline 50.0 & 12.0 & - & 20.0 & 4.0 & & & & & & (14.0 & & ; & 76.3 & \\
\hline 50.0 & 10.0 & & 15.0 & 5.0 & & & & & & $(20.0$ & & ; & 78.2 & \\
\hline 50.0 & 10.0 & & 10.0 & 5.0 & & & & & & $(25.0$ & & 's & 79.5 & \\
\hline 18.6 & 19.7 & 16.9 & 5.1 & & & & & & & $(40.0$ & & s & 76.8 & \\
\hline & & & 45.0 & $10 . n$ & & $(25.0$ & 1 & & & $(20.0$ & & f & 77.0 & \\
\hline 60.0 & & & i11.0 & & 20.0 & & & & & )$(10.0$ & & ? & 77.7 & \\
\hline 44.0 & 16.0 & & & & $\left\{\begin{array}{l}12.0 \\
0\end{array}\right.$ & & & & . & $\langle 2 B: 0$ & & , & 778 & \\
\hline & 50.0 & & 5.0 & 10.0 & $(20.0$ & & . & & & $\{(15.0$ & & ) & 79.1 & . \\
\hline & 45.0 & & & 15.0 & $20: 0$ & & & & & 20.0 & & 1 & 79.5 & \\
\hline 20.0 & 40.0 & & & 5.0 & 10.0 & & 5.0 & & 10.0 & 10.0 & & & 78.5 & \\
\hline & & & & 160.0 & & & 125.0 & & & )$(15.0$ & & ) & 77.4 & \\
\hline & & & & 165.0 & & & (25.0 & & & )$(10.0$ & & j & 79.6 & \\
\hline & & & & $(70.0$ & & & $(20.0$ & & & )$(10.0$ & & ) & 74.4 & \\
\hline & & & & $(70.0$ & & & (15.0 & & & $\{15.0$ & & ? & 75.9 & \\
\hline & & & & $(60.0$ & & & (15.0 & & & )$(25.0$ & & j & 75.9 & \\
\hline & & & & 65.0 & & & $(10.0$ & & & $(25.0$ & & j & 76.3 & \\
\hline & & & & 67.5 & & & 20.0 & & & )$(12.5$ & & $j$ & 76.6 & \\
\hline & & & & 65.0 & i & & $(20.0$ & & & )$(1.5 .0$ & & ) & 76.8 & \\
\hline & & & & $(75.0$ & j & & & & & 125.0 & & j & 75.5 & \\
\hline & 30.0 & 30.0 & & & & & 15.0 & & & 25.0 & & & 78.5 & \\
\hline 48.4 & & & & & & 26.6 & & & & 25.0 & & & 76.4 & \\
\hline & & & & $(60.0$ & & & $(30.0$. & & & )$(10.0$ & & ) & 76.4 & \\
\hline & & & & 175.0 & & & & & & $(25.0$ & & f & 76.2 & \\
\hline 50.0 & & & & & & & & & & 50.0 & & ? & 78.2 & \\
\hline & 40.0 & 40.0 & & & & & & & & $(20.0$ & & f' & 75.3 & \\
\hline & 30.0 & & & & & & 40.0 & & & 30.0 & & j & 79.0 & \\
\hline & & & 60.0 & & & & & & & $(40.0$ & & ? & 77.7 & \\
\hline & & & 50.0 & & & & & & & 50.0 & & ) & 78.6 & \\
\hline & & & 70.0 & & & & & & & 30.0 & & ; & 19.0 & \\
\hline 60.0 & & & & & & 30.0 & & & & (10.0 & & $j$ & 79.6 & \\
\hline 80.0 & & & & & & & & & & $(20.0$ & & j & 77.9 & \\
\hline 45.0 & & & & & & & & & 35.0 & & & $20.0^{\circ}$ & 77.1 & \\
\hline & & & 70.0 & & & & & 30.0 & & & & & 70.6 & \\
\hline & & 34.6 & & & . & & 52.6 & & & $(12.8$ & & ) & 75.1 & \\
\hline 60.9 & & 9.1 & & & & 30.0 & & . & & & & & 77.0 & \\
\hline 90.0 & 10.0 & & & & & 30.0 & & & & & & & 75.1 & \\
\hline 56.0 & 14.0 & & & & & 30.0 & & & & & & & 77.9 & \\
\hline 49.0 & $21: 0$ & & & & & $3 n . n$ & & & & & & & 75.7 & \\
\hline 42.0 & 28.0 & & & & & 30.0 & & & & & & & 80.4 & \\
\hline 28.0 & 42.0 & & & & & 30.0 & & & & & & & 75.5 & \\
\hline
\end{tabular}




\section{REFERENCES}

1. McGeary, R. K., J. Am. Ceramic Soc., L4, 513-522, 1961.

2. Westman, A. E. R., and Hugill, H. R., I. Am. Ceramic Soc., 13, 767$779,1930$.

3. Hauth, J. J., "Vibrationally Compacted Ceramic Fuels," HW-67777, January, 1961.

4. CEND-152, "The Development and Testing of UO Fuel Systems for Water Reactor Applications," Second Quarterly Report, Oct. - Dec., 1961. 